\title{
A Class of Nonzero-Sum Stochastic Differential Investment and Reinsurance Games
}

\author{
Alain Bensoussan $^{*, a}$, Chi Chung Siu ${ }^{\dagger, b}$, Phillip Yam ${ }^{\ddagger, c}$, and Hailiang \\ Yang $\S, \mathrm{d}$ \\ ${ }^{a}$ International Center for Decision and Risk Analysis, School of Management, The University of \\ Texas at Dallas, \\ Richardson, TX, USA. \\ ${ }^{a}$ Department of Systems Engineering and Engineering Management, College of Science and \\ Engineering, City University of Hong Kong, \\ Hong Kong, China \\ ${ }^{a}$ Graduate Department of Financial Engineering, Ajou University, \\ Suwon, South Korea \\ ${ }^{\mathrm{b}}$ School of Business, The University of Technology, Sydney, \\ NSW, Australia. \\ ${ }^{\mathrm{c}}$ Department of Statistics, The Chinese University of Hong Kong, \\ Shatin, N.T., Hong Kong, China \\ ${ }^{\mathrm{d}}$ Department of Statistics and Actuarial Science, The University of Hong Kong, \\ Pokfulam Road, Hong Kong, China
}

\begin{abstract}
In this article, we provide a systematic study on the nonzero-sum stochastic differential investment and reinsurance game between two insurance companies. Each insurance company's surplus process consists of a proportional reinsurance protection and an investment in risky and risk-free assets. Each insurance company is assumed to maximize his utility of the difference between his terminal surplus and that of his competitor. The surplus process of each insurance company is modeled by a mixed regime-switching Cramer-Lundberg diffusion approximation process, i.e. the coefficients of the diffusion risk processes are modulated by a continuous-time Markov chain and an independent market-index process. Correlation between the two surplus processes, independent of the risky asset process, is allowed. Despite the complex structure, we manage to solve the resulting non-zero sum game problem by applying the dynamic programming principle. The Nash equilibrium, the optimal reinsurance/investment, and the resulting value processes of the insurance companies are obtained in closed forms, together with sound economic interpretations, for the case of an exponential utility function.
\end{abstract}

\footnotetext{
*Email: axb046100@utdallas.edu

†Email: chichung.siu@uts.edu.au

${ }^{\ddagger}$ Email: scpyam@sta.cuhk.edu.hk

$\S$ Email: hlyang@hku.hk
} 
Keywords: Hamiltonian-Jacobi-Bellman equation; Nonzero-sum stochastic differential game; Equilibrium investment; Equilibrium proportional reinsurance; Regime-switching models; Relative performance; Cramer-Lundberg model; Nash equilibrium; stochastic control.

\section{Introduction}

Since the classical works of Bühlmann (1970) and Gerber (1970), the study of optimal investment and reinsurance strategies has been a recurrent theme in the insurance literature. In practice, insurance companies can diversify their risks by purchasing reinsurance protection from a reinsurance company, and also by investing in the stock markets. For example, Browne (1995) validated the long-standing conjecture concerning the relation between minimizing the probability of ruin and maximizing the exponential utility. More specifically, when there is no interest rate, Browne (1995) proved the equivalence between a policy that maximizes the exponential utility function of the wealth at a fixed terminal time and a policy that minimizes the probability of ruin. Yang and Zhang (2005) obtained the closed-form solution to the optimal investment policies of an insurer under a jump-diffusion risk process. Liu and Ma (2009) revisited the optimal reinsurance/investment/consumption problem under a general insurance risk model. More specifically, by modeling the reserve process of the insurance company using a stochastic differential equation (SDE) driven by a Brownian motion and a Poisson random measure, they modified the "duality method" in finance and the solvability of a special type of backward stochastic differential equation (BSDE) to obtain the necessary and sufficient conditions for the well-posedness and solvability of the utility maximization problems.

Over the past decades, the theory of stochastic differential games has been studied extensively in the realm of control theory. At the theoretical level, many fundamental results on the existence of Nash equilibria have been obtained. For example, Elliott (1976) examined the relationship between the values of the zero-sum stochastic differential games with two players and the Isaacs condition. Regarding the development of the non-zero sum stochastic differential games, the pioneering work by one of our authors (Bensoussan and Frehse (2000)) used the dynamic programming approach to solve the non-zero sum stochastic differential game with $N$ players over an infinite time horizon. They showed that the existence of the Nash equilibrium can be proved by applying the regularity theory of the system of nonlinear partial differential equations (PDEs).

In the contexts of finance and insurance, there has been rapid development in extending the optimal investment/reinsurance problem in the context of stochastic differential games. Browne (2000) studied the zero-sum stochastic differential games of two competing investors with one payoff function depending on both investors' wealth processes. Recently, Zeng (2010) studied the existence of the Nash equilibrium in a zero-sum stochastic differential reinsurance game. Øksendal and Sulem (2011) formulated the model uncertainty of the optimal portfolio problem in the form of a zero-sum game. Elliott and Siu (2011a) adopted the BSDE approach to study a similar zero-sum game between the insurer and the market, in which the insurer aims to minimize the risk described by a convex risk measure of his terminal wealth, whereas the market selects the probability measure that maximizes the risk under the worst-case scenario. 
Economic trends often have a significant influence on the dynamics of asset prices. Correspondingly, the optimal reinsurance/investment strategies should be subject to changes in the economic trends. However, the aforementioned works studied the non-game or game problem under the assumption that the reserve process follows a jump-diffusion process with constant coefficients. To incorporate changes in economic trends, Markovian regime-switching processes, i.e. the SDEs with coefficients modulated by the continuous-time Markov chain, prove to be a valuable class of candidate processes with tractable properties. For example, Elliott et al. (2010) developed the robust filter-based and smoother-based ExpectationMaximization (EM) algorithms to recursively estimate the unknown parameters of insurance claim processes when the arrival and size of the claims are state-dependent. In earlier works by one of our authors, Ng and Yang (2006) and Zhu and Yang (2009) studied the joint distribution of the surplus process immediately before/after the ruin and the differentiability of the ruin functions under the Markovian regime-switching environment, respectively. To further develop the control theory in finance and insurance, Zhou and Yin (2003) considered the mean-variance problem under the regime-switching diffusion process, which Chen and Yam (2013) recently extended to the case of reinsurance and investment strategies with short-sell prohibition. Zhu (2011) derived the optimal control that minimizes the total cost of an insurance company up to a stochastic exit time under the regime-switching diffusion framework. In the context of stochastic differential games, Elliott and Siu (2011b) formulated the optimal investment problem in the form of a zero-sum stochastic differential games between the insurance company and the market. They adopted a Girsanov transform for the regime-switching Markov chain to incorporate the model risk when modeling the economic risk introduced by the Markov chain.

The development of non-zero sum stochastic differential games in finance and insurance is relatively new. Espinosa and Touzi (2013) considered the problem of optimal investment when agents take into account their relative performance against their peers in the form of a non-zero sum stochastic differential game with $N$ players. They showed the existence and uniqueness of the Nash equilibrium under the cases of unconstrained and constrained agents with exponential utilities within the Black-Scholes market framework, i.e. with constant coefficients in the corresponding SDEs. Wang and Yu (2012) studied a specific form of the non-zero sum differential game under the BSDE framework. In particular, they established a necessary Pontryagin maximum principle for an open-loop Nash equilibrium point of a partial information game and provided a sufficient condition for the existence of the Nash equilibrium point.

In this paper, we study the non-zero sum reinsurance and investment game in the context of mixed regime-switching reserve processes, in which the correlation between the competing insurance companies is allowed. We formulate the problem via the dynamic programming principle, resulting in the coupled Hamilton-Jacobi-Bellman (HJB) formulation under a general, differentiable, concave utility function with regime-switching effects. We connect the existence of the Nash equilibrium to the solvability of the coupled system of nonlinear PDEs. For the case of the exponential utility function, we obtain explicit solutions for the reinsurance/investment strategies of two competing insurance companies and the corresponding value functions at equilibrium, together with economic interpretations.

The rest of the paper is organized as follows. Section 2 defines the risk and surplus processes of each competitive insurance company and his reinsurance/investment opportunities. Section 3 provides the objective of each insurer. We assume that the prime objective of each insurer 
is to maximize the expected terminal utility of the difference between his surplus process and that of his competitor. Section 4 formulates the non-zero sum stochastic differential game problem by means of the dynamic programming principle. The conditions for the existence of the Nash equilibrium and the verification theorem for the corresponding (coupled) HJB equations are provided. In Section 5, we provide the explicit solutions for the equilibrium reinsurance/investment strategies under the case of the exponential utility function with some numerical illustrations. Section 6 concludes the paper with suggestions for future research.

\section{Model}

Let $(\Omega, \mathcal{F}, \mathbb{P})$ be a complete probability space and $\left[0, T^{*}\right]$ be the planning horizon, with $0<T^{*}<\infty$. Here, we assume that $\mathcal{F}=\left(\mathcal{F}_{t}\right)_{t \geq 0}$, where $\mathcal{F}_{t} \triangleq \mathcal{F}_{t}^{\alpha} \vee \mathcal{F}_{t}^{B}$. Processes $\{\alpha(t)\}_{t \geq 0}$ and $\{B(t)\}_{t \geq 0} \triangleq\left\{\left(B_{1}(t), B_{2}(t), B_{L}(t), B_{S}(t)\right)\right\}_{t \geq 0}$, are defined.

\subsection{Dynamics of economic trends}

Let $E=\{1, \ldots, d\}$ be the finite state space. Following Elliott and Siu (2011b) and the references therein, we model the state of the economy over time by the continuous time, finite state, observable Markov chain, $\{\alpha(t)\}_{t \geq 0}$, taking the values in the state space $E$.

To specify the distributional properties of the Markov chain $\{\alpha(t)\}_{t \geq 0}$, let $\mathbf{Q}=\left(q_{i j}\right)_{i, j \in E}$ denote the Q-matrix of $\{\alpha(t)\}_{t \geq 0}$, such that $q_{i j} \geq 0$, for $j \neq i, q_{i i}=-\sum_{j \neq i} q_{i j} \leq 0$.

According to Elliott et al. (1994), the Markov chain $\{\alpha(t)\}_{t \geq 0}$ admits the following semimartingale representation:

$$
\alpha(t)=\alpha(0)+\int_{0}^{t} \mathbf{Q}^{\top} \alpha(u) \mathrm{d} u+M(t), t>0
$$

where $M(t)$ is some $\mathbb{R}^{d}$-valued martingale with respect to filtration $\left(\mathcal{F}^{\alpha}\right)_{t \geq 0}$, generated by $\{\alpha(t)\}_{t \geq 0}$. Finally, $\mathbf{b}^{\top}$ denotes the transpose of $\mathbf{b}$.

\subsection{Market index process}

In addition to the changes in economic trends modeled by the Markov chain $\{\alpha(t)\}_{t \geq 0}$, we assume there is another diffusion process, independent of the Markov chain $\{\alpha(t)\}_{t \geq 0}$, that captures the dynamics of the market index. Let $\{L(t)\}_{t \geq 0}$ denote a market index process with the following representation

$$
\mathrm{d} L(t)=\mu_{L}(t, L(t)) \mathrm{d} t+\sigma_{L}(t, L(t)) \mathrm{d} B_{L}(t),
$$

where $\left\{B_{L}(t)\right\}_{t \geq 0}$ is the standard Brownian motion independent of $\left\{B_{1}(t)\right\}_{t \geq 0},\left\{B_{2}(t)\right\}_{t \geq 0}$, and $\left\{B_{S}(t)\right\}_{t \geq 0}$, the Brownian motions to be defined below. Let $\left(\mathcal{F}_{t}^{B_{L}}\right)_{t \geq 0}$ denote the filtration generated by Brownian motion $\left\{B_{L}(t)\right\}_{t \geq 0}$.

A common model of the market index process includes a mean-reverted diffusion process such as the Ornstein-Uhlenbeck process, where $\mu_{L}(t, L(t))$ is an affine function of $L(t)$ and $\sigma_{L}$ is a 
constant. To ensure the SDE in (2) admits a unique strong solution, we assume that $\mu_{L}$ and $\sigma_{L}$ satisfy a uniform Lipschitz condition, i.e. there exists $K_{L} \geq 0$ for all $l_{1}, l_{2} \in \mathbb{R}$, such that

$$
\left|\mu_{L}\left(., l_{1}\right)-\mu_{L}\left(., l_{2}\right)\right|+\left|\sigma_{L}\left(., l_{1}\right)-\sigma_{L}\left(., l_{2}\right)\right| \leq K_{L}\left|l_{1}-l_{2}\right| .
$$

In this case, Doob's $L^{p}$-inequality gives

$$
\mathbb{E}\left[\sup _{0 \leq t \leq T^{*}}|L(t)|^{2}\right]<\infty
$$

\section{$2.3 \quad$ Insurance companies}

Consider a market with two competing insurance companies and one reinsurance company. Following the assumption that the companies have large insurance portfolios relative to the size of each individual claim, we model the surplus process of each insurer using the standard Cramer-Lundberg diffusion approximation. See, for example, Klugman et al. (2008), for the standard treatise on the Cramer-Lundberg diffusion approximation in insurance models. More specifically, $\left\{R_{k}(t)\right\}_{t \geq 0}$ denotes the Cramer-Lundberg diffusion approximation of the surplus process of insurer $k \in\{1,2\}$, then $\left\{R_{k}(t)\right\}_{t \geq 0}$ takes the form

$$
\left.\mathrm{d} R_{k}(t)=p_{k}(\alpha(t), L(t))-\lambda_{k}(\alpha(t), L(t)) \mathbb{E}\left[\xi_{k}\right] \mathrm{d} t+\sqrt{\lambda_{k}(\alpha(t), L(t)) \mathbb{E}\left[\xi_{k}^{2}\right.}\right] \mathrm{d} B_{k}(t),
$$

where $p_{k}>0$ is the uniformly bounded premium rate, $\lambda_{k}>0$ is a uniformly bounded arrivalrate function of claims, and $\xi_{k} \neq 0$ is a random variable representing the size of the claims with $\mathbb{E}\left[\xi_{k}^{2}\right]<\infty$, for $k=1,2$. $\left(\mathcal{F}_{t}^{B_{k}}\right)_{t \geq 0}$, for $k=1,2$, denotes the filtration generated by the standard Brownian motion $\left\{B_{k}(t)\right\}_{t \geq 0}$.

To capture the notion that these two competing insurers have negligible effects on the economy, we assume that $\left\{B_{k}(t)\right\}_{t \geq 0}$ and $\{\alpha(t)\}_{t \geq 0}$ are mutually independent, for $k=1$, 2. To model the dependence between two insurance companies, we assume that $\left\{B_{1}(t)\right\}_{t \geq 0}$ and $\left\{B_{2}(t)\right\}_{t \geq 0}$ are correlated, i.e. $\mathrm{d}\left\langle B_{1}(t), B_{2}(t)\right\rangle=\rho \mathrm{d} t$, for $\rho \in[-1,1]$.

\subsection{Reinsurance and investment opportunities}

To reduce the underlying risks, each insurer has an option to purchase a proportional reinsurance protection. Let $\theta_{k}$ be the premium rate paid by insurer $k \in\{1,2\}$ to the reinsurance company. Let $\left\{a_{k}(t)\right\}_{t \geq 0}$ be the $\mathcal{F}$-progressively measurable process valued in [0,1], representing the proportional reinsurance protection bought by insurer $k \in\{1,2\}$ at $t \geq 0$. Let $A_{k} \triangleq[0,1]$ be the set of proportional reinsurance strategies of insurer $k^{1}{ }^{1}$ In other words, upon purchasing the proportional reinsurance protection, the reinsurance company will cover $\left(1-a_{k}(t)\right) 100 \%$ of the claims, while insurance company $k$ will cover the remaining $a_{k}(t) \%$.

After purchasing reinsurance, the surplus process of insurer $k \in\{1,2\}$, denoted as $\left\{R_{k}^{a_{k}}(t)\right\}_{t \geq 0}$, becomes

$$
\begin{aligned}
\mathrm{d} R_{k}^{a_{k}}(t) \triangleq & {\left[p_{k}(\alpha(t), L(t))-a_{k}(t) \lambda_{k}(\alpha(t), L(t)) \mathbb{E}\left[\xi_{k}\right]-\left(1-a_{k}(t)\right) \theta_{k}(\alpha(t), L(t))\right] \mathrm{d} t } \\
& +a_{k}(t) \sqrt{\lambda_{k}(\alpha(t), L(t)) \mathbb{E}\left[\xi_{k}^{2}\right]} \mathrm{d} B_{k}(t),
\end{aligned}
$$

\footnotetext{
${ }^{1}$ The same method can be applied to the case when $A_{k} \subset[0,1]$, for $k \in\{1,2\}$.
} 
where the premium rate of the proportional reinsurance protection, $\theta_{k}(\alpha(t), L(t))>0$, is assumed to be uniformly bounded.

For simplicity, we write $R_{k}^{a_{k}}(t)$ as follows:

$$
\mathrm{d} R_{k}^{a_{k}}(t)=\left(\mu_{k}(\alpha(t), L(t))+\bar{\theta}_{k}(\alpha(t), L(t)) a_{k}(t)\right) \mathrm{d} t+a_{k}(t) \sigma_{k}(\alpha(t), L(t)) \mathrm{d} B_{k}(t),
$$

where

$$
\begin{aligned}
\mu_{k}(\alpha(t), L(t)) & \triangleq p_{k}(\alpha(t), L(t))-\theta_{k}(\alpha(t), L(t)), \\
\bar{\theta}_{k}(\alpha(t), L(t)) & \triangleq \theta_{k}(\alpha(t), L(t))-\lambda_{k}(\alpha(t), L(t)) \mathbb{E}\left[\xi_{k}\right], \\
\sigma_{k}(\alpha(t), L(t)) & \triangleq \sqrt{\lambda_{k}(\alpha(t), L(t)) \mathbb{E}\left[\xi_{k}^{2}\right]} .
\end{aligned}
$$

Note that the uniform boundedness of $p_{k}, \theta_{k}$, and $\lambda_{k}$, together with the finiteness of $\mathbb{E}\left[\xi_{k}^{2}\right]$, ensure that $\mu_{k}, \bar{\theta}_{k}$, and $\sigma_{k}$ are uniformly bounded in $\mathbb{R}$, for $k=1,2$.

In addition to purchasing reinsurance protection, assume that each insurer $k$, for $k=1,2$, can invest in a risk-free asset, denoted as $\left\{S_{0}(t)\right\}_{t \geq 0}$, and a risky asset, denoted as $\{S(t)\}_{t \geq 0}$. The dynamics of the risk-free asset $\left\{S_{t}^{0}\right\}_{t \geq 0}$ are given as

$$
\frac{\mathrm{d} S_{0}(t)}{S_{0}(t)}=r \mathrm{~d} t
$$

where $r>0$ denotes the constant, risk-free interest rate.

The dynamics of the risky asset $\{S(t)\}_{t \geq 0}$ are given as

$$
\frac{\mathrm{d} S(t)}{S(t)}=m_{S}(\alpha(t), L(t)) \mathrm{d} t+\sigma_{S}(\alpha(t), L(t)) \mathrm{d} B_{S}(t)
$$

where $m_{S}$ and $\sigma_{S}>0$ denote the uniformly bounded return and the volatility functions of the risky asset $S$, respectively. $\left(\mathcal{F}_{t}^{B_{S}}\right)_{t \geq 0}$ denotes the filtration generated by the standard Brownian motion $\left\{B_{S}(t)\right\}_{t \geq 0}$. We assume that $\left\{B_{k}(t) \mid t \in\left[0, T^{*}\right], k=1,2\right\}$ and $\left\{B_{S}(t) \mid t \in\right.$ $\left.\left[0, T^{*}\right]\right\}$ are independent. ${ }^{2}$

Let $\left\{b_{k}(t)\right\}_{t \geq 0}$ be an $\mathcal{F}$-progressively measurable process valued in $\mathbb{R}$ representing the amount that insurer $k$ invests in the risky asset $S$ at $t \geq 0$. Here, we assume that short-selling of the risky asset is allowed. Let $B_{k}$ denote the set of investment strategies of insurer $k$. Let $\left\{X_{k}^{\pi_{k}}(t)\right\}_{t \geq 0}$, where $\pi_{k}(t) \triangleq\left(a_{k}(t), b_{k}(t)\right) \in A_{k} \times B_{k}$, be the surplus process of insurer $k$ after purchasing reinsurance protection $\left(a_{k}\right)$ and making investment $\left(b_{k}\right)$. Following the common approach to the proportional reinsurance and investment models (see, for example, Liu and Ma (2009) and Chen and Yam (2013)), insurer $k$ 's surplus process can be modeled as follows

$$
\begin{aligned}
\mathrm{d} X_{k}^{\pi_{k}}(t)= & {\left[r X_{k}^{\pi_{k}}(t)+\mu_{k}(\alpha(t), L(t))+\bar{\theta}_{k}(\alpha(t), L(t)) a_{k}(t)\right.} \\
& \left.+b_{k}(t)\left(m_{S}(\alpha(t), L(t))-r\right)\right] \mathrm{d} t \\
& +a_{k}(t) \sigma_{k}(\alpha(t), L(t)) \mathrm{d} B_{k}(t)+b_{k}(t) \sigma_{S}(\alpha(t), L(t)) \mathrm{d} B_{S}(t), \quad k=1,2
\end{aligned}
$$

\footnotetext{
${ }^{2}$ As we assume the two competing insurers have no influence on the market, their investment decisions should have no effect on the stock market. The independence between $\left\{B_{k}(t) \mid t \in\left[0, T^{*}\right], k=1,2\right\}$ and $\left\{B_{S}(t) \mid t \in\left[0, T^{*}\right]\right\}$ reflects this assumption.
} 
Observe that the processes $\left\{\mu_{k}(\alpha(t), L(t))\right\}_{t \geq 0},\left\{\sigma_{k}(\alpha(t), L(t))\right\}_{t \geq 0},\left\{m_{S}(\alpha(t), L(t))\right\}_{t \geq 0}$, and $\left\{\sigma_{S}(\alpha(t), L(t))\right\}_{t \geq 0}$ are $\left(\mathcal{F}^{\alpha} \vee \mathcal{F}^{B_{L}}\right)_{t \geq 0}$-adapted. To ensure that the SDE in (10) admits a strong unique solution, we impose additional assumptions on the strategy $\left(a_{k}, b_{k}\right)$ of insurer $k \in\{1,2\}$. To this end, let us first denote, for $k=1,2$,

$$
\begin{gathered}
d_{k}\left(x, l, j, a_{k}, b_{k}\right) \triangleq r x+\mu_{k}(j, l)+\bar{\theta}_{k} a_{k}+b_{k}\left(m_{S}(j, l)-r\right), \\
v_{k}\left(x, l, j, a_{k}, b_{k}\right) \triangleq a_{k} \sigma_{k}(j, l), \quad v_{k}^{S}\left(x, l, j, a_{k}, b_{k}\right) \triangleq b_{k} \sigma_{S}(j, l) .
\end{gathered}
$$

Let $\mathcal{A}_{k} \subset A_{k}$ and $\mathcal{B}_{k} \subset B_{k}$ be the set of strategies $\left(a_{k}, b_{k}\right)$ of insurer $k$ such that $d_{k}$, $v_{k}$, and $v_{k}^{S}$ satisfy the following.

1. A uniformly Lipschitz condition in $\mathcal{A}_{k} \times \mathcal{B}_{k}$ :

there exists $K \geq 0$, such that for all $x, y, l_{1}, l_{2} \in \mathbb{R}$ and $\left(a_{k}, b_{k}\right) \in \mathcal{A}_{k} \times \mathcal{B}_{k}$,

$$
\begin{aligned}
& \sup _{j \in E}\left\{\left|d_{k}\left(x, l_{1}, j, a_{k}, b_{k}\right)-d_{k}\left(y, l_{2}, j, a_{k}, b_{k}\right)\right|+\left|v_{k}\left(x, l_{1}, j, a_{k}, b_{k}\right)-v_{k}\left(y, l_{2}, j, a_{k}, b_{k}\right)\right|\right. \\
& \left.\quad+\left|v_{k}^{S}\left(x, l_{1}, j, a_{k}, b_{k}\right)-v_{k}^{S}\left(y, l_{2}, j, a_{k}, b_{k}\right)\right|\right\} \leq K\left(|x-y|+\left|l_{1}-l_{2}\right|\right) .
\end{aligned}
$$

2. $L^{2}$-integrability condition in $\mathcal{A}_{k} \times \mathcal{B}_{k}$ :

for all $x, l \in \mathbb{R}$ and $\left(a_{k}, b_{k}\right) \in \mathcal{A}_{k} \times \mathcal{B}_{k}$,

$$
\begin{aligned}
\sup _{j \in E} \mathbb{E}\left\{\int _ { 0 } ^ { T ^ { * } } \left[\left|d_{k}\left(x, l, j, a_{k}(t), b_{k}(t)\right)\right|^{2}\right.\right. \\
\left.\left.\quad+\left|v_{k}\left(x, l, j, a_{k}(t), b_{k}(t)\right)\right|^{2}+\left|v_{k}^{S}\left(x, l, j, a_{k}(t), b_{k}(t)\right)\right|^{2}\right] \mathrm{~d} t\right\}<\infty .
\end{aligned}
$$

We use $\Pi_{k} \triangleq \mathcal{A}_{k} \times \mathcal{B}_{k}$ to denote the set of strategies $\pi_{k}=\left(a_{k}, b_{k}\right) \in \Pi_{k}$ of insurer $k \in\{1,2\}$, satisfying (11) and (12).

From the standard stochastic control theory (see, for example, Pham (2009)), conditions (11) and (12) ensure that for all $\pi_{k}=\left(a_{k}, b_{k}\right) \in \Pi_{k}$, and for any initial condition $(t, x, l, i) \in$ $\left[0, T^{*}\right] \times \mathbb{R} \times \mathbb{R} \times E$, the $\mathrm{SDE}$ in (10) admits a strong unique solution. In this case, we also have

$$
\mathbb{E}\left[\sup _{0 \leq t \leq T^{*}}\left|X_{k}^{\pi_{k}}(t)\right|^{2}\right]<\infty, \quad \text { for } \quad k=1,2 .
$$

\section{Objectives of the insurance companies}

Insurer $k=1,2$ has a utility function denoted as $U_{k}: \mathbb{R} \rightarrow \mathbb{R}$, where $U_{k}$ is assumed to be increasing, strictly concave, and satisfies Inada conditions, i.e.,

$$
\partial_{x} U_{k}(-\infty)=+\infty, \quad \partial_{x} U_{k}(+\infty)=0 .
$$


Similar to Espinosa and Touzi (2013), we assume that the prime objective of insurer $k$ is to maximize the expected utility of his performance at the terminal time $T \in\left[0, T^{*}\right)$, relative to his competitor. That is, insurer $k$ will choose a reinsurance/investment strategy $\pi_{k}=\left(a_{k}, b_{k}\right) \in \Pi_{k}$ such that

$$
\mathbb{E}\left[U_{k}\left(\left(1-\kappa_{k}\right) X_{k}^{\pi_{k}}(T)+\kappa_{k}\left(X_{k}^{\pi_{k}}(T)-X_{m}^{\pi_{m}}(T)\right)\right)\right]=\mathbb{E}\left[U_{k}\left(X_{k}^{\pi_{k}}(T)-\kappa_{k} X_{m}^{\pi_{m}}(T)\right)\right],
$$

for $m \neq k \in\{1,2\}$, is maximized. Here, $\kappa_{k} \in[0,1], k=1,2$, measures the sensitivity of insurer $k$ to the performance of his competitor.

In other words,

Problem 3.1. Find a Nash equilibrium $\left(\pi_{1}^{*}, \pi_{2}^{*}\right) \in \Pi_{1} \times \Pi_{2}$ such that

$$
\left\{\begin{array}{l}
\mathbb{E}\left[U_{1}\left(X_{1}^{\pi_{1}}(T)-\kappa_{1} X_{2}^{\pi_{2}^{*}}(T)\right)\right] \leq \mathbb{E}\left[U_{1}\left(X_{1}^{\pi_{1}^{*}}(T)-\kappa_{1} X_{2}^{\pi_{2}^{*}}(T)\right)\right] \\
\mathbb{E}\left[U_{2}\left(X_{2}^{\pi_{2}}(T)-\kappa_{2} X_{1}^{\pi_{1}^{*}}(T)\right)\right] \leq \mathbb{E}\left[U_{2}\left(X_{2}^{\pi_{2}^{*}}(T)-\kappa_{2} X_{1}^{\pi_{1}^{*}}(T)\right)\right]
\end{array}\right.
$$

\section{Nash Equilibrium}

To establish a Nash equilibrium for Problem 3.1, we study the problem in the form of a dynamic programming principle. For notational convenience, we suppress the arguments of the functions of $\alpha(t)$ and $L(t)$. For example, we abbreviate $m_{S} \triangleq m_{S}(\alpha(t), L(t))$. To this end, observe first that, for $m \neq k \in\{1,2\}$,

$$
\begin{aligned}
\mathrm{d} X_{m}^{\pi_{m}}(t)= & {\left[r X_{m}^{\pi_{m}}(t)+\mu_{m}+\bar{\theta}_{m} a_{m}(t)+b_{m}(t)\left(m_{S}-r\right)\right] \mathrm{d} t } \\
& +a_{m}(t) \sigma_{m} \mathrm{~d} B_{m}(t)+b_{m}(t) \sigma_{S} \mathrm{~d} B_{S}(t),
\end{aligned}
$$

hence, it readily follows that

$$
\begin{aligned}
\mathrm{d}\left(X_{k}^{\pi_{k}}-\kappa_{k} X_{m}^{\pi_{m}}\right)(t)= & {\left[r\left(X_{k}^{\pi_{k}}(t)-\kappa_{k} X_{m}^{\pi_{m}}(t)\right)+\left(\mu_{k}-\kappa_{k} \mu_{m}\right)+\left(\bar{\theta}_{k} a_{k}(t)-\kappa_{k} \bar{\theta}_{m} a_{m}(t)\right)\right.} \\
& \left.+\left(b_{k}(t)-\kappa_{k} b_{m}(t)\right)\left(m_{S}-r\right)\right] \mathrm{d} t+a_{k}(t) \sigma_{k} \mathrm{~d} B_{k}(t)-\kappa_{k} a_{m}(t) \sigma_{m} \mathrm{~d} B_{m}(t) \\
& +\left(b_{k}(t)-\kappa_{k} b_{m}(t)\right) \sigma_{S} \mathrm{~d} B_{S}(t) .
\end{aligned}
$$

Let

$$
V^{k}(t, x, l, i) \triangleq \sup _{\pi_{k} \in \Pi_{k}} \mathbb{E}\left[U\left(X_{k}^{\pi_{k}}(T)-\kappa_{k} X_{m}^{\pi_{m}^{*}}(T)\right)\right], \quad \text { for } \quad k \neq m \in\{1,2\}
$$

be the value function in $\mathbb{R}_{+} \times \mathbb{R} \times \mathbb{R} \times E$. Denote $X_{k}^{\pi_{k}}(t) \triangleq x_{k}, a_{k} \triangleq a_{k}(t)$, and $b_{k} \triangleq b_{k}(t)$, for $k=1,2$.

\subsection{Verification Theorem}

Following the standard ideas in the dynamic programming principle (see Pham (2009)), the verification-type theorem below presents the first important result of the present work. 
Theorem 4.1. (Verification Theorem)

Denote $\Gamma \triangleq\left[0, T^{*}\right) \times \mathbb{R} \times \mathbb{R} \times E$ and $\bar{\Gamma} \triangleq\left[0, T^{*}\right] \times \mathbb{R} \times \mathbb{R} \times E$.

Let $W^{k}$ be a function in $\mathcal{C}^{1,2}(\Gamma) \cap \mathcal{C}^{0}(\bar{\Gamma})$ that satisfies a quadratic growth condition, i.e. there exists $C>0$ such that

$$
\left|W^{k}(t, x, l, j)\right| \leq C\left(1+|x|^{2}+|l|^{2}\right), \quad \text { for all }(t, x, l, j) \in \bar{\Gamma}, \quad k=1,2 .
$$

Define, for $x \triangleq x_{k}-\kappa_{k} x_{m}$, for $m \neq k \in\{1,2\}$,

$$
\begin{aligned}
\mathcal{L}^{\pi_{k}} W^{k}(t, x, l, i) \triangleq & {\left[r x+\left(\mu_{k}(i, l)-\kappa_{k} \mu_{m}(i, l)\right)+\left(\bar{\theta}_{k}(i, l) a_{k}(t)-\kappa_{k} \bar{\theta}_{m}(i, l) a_{m}^{*}(t)\right)\right.} \\
& \left.+\left(b_{k}(t)-\kappa_{k} b_{m}^{*}(t)\right)\left(m_{S}(i, l)-r\right)\right] W_{x}^{k}(t, x, l, i) \\
& +\frac{1}{2}\left[\left(a_{k}^{2}(t) \sigma_{k}^{2}(i, l)-2 a_{k}(t) a_{m}^{*}(t) \kappa_{k} \sigma_{k}(i, l) \sigma_{m}(i, l) \rho+\kappa_{k}^{2} a_{m}^{* 2}(t) \sigma_{m}^{2}(i, l)\right)\right. \\
& \left.+\left(b_{k}^{2}(t)-2 \kappa_{k} b_{k}(t) b_{m}^{*}(t)+\kappa_{k}^{2} b_{m}^{* 2}(t)\right) \sigma_{S}^{2}(i, l)\right] W_{x x}^{k}(t, x, l, i) \\
& +\mu_{L}(t, l) W_{l}^{k}(t, x, l, i)+\frac{1}{2} \sigma_{L}^{2}(t, l) W_{l l}^{k}(t, x, l, i) \\
& +\sum_{j \in E} q_{i j} W^{k}(t, x, l, j) .
\end{aligned}
$$

and define $\pi_{k}^{*} \triangleq\left(a_{k}^{*}, b_{k}^{*}\right)$, where

$$
\begin{aligned}
a_{k}^{*}(t)= & \underset{a_{k}(t) \in \mathcal{A}_{k}}{\arg \max }\left\{\left[\bar{\theta}_{k}(i, l) a_{k}(t)\right] W_{x}^{k}(t, x, l, i)\right. \\
& \left.+\frac{1}{2}\left[a_{k}^{2}(t) \sigma_{k}^{2}(i, l)-2 \kappa_{k} a_{k}(t) a_{m}^{*}(t) \sigma_{k}(i, l) \sigma_{m}(i, l) \rho\right] W_{x x}^{k}(t, x, l, i)\right\} \\
b_{k}^{*}(t)= & \underset{b_{k}(t) \in \mathcal{B}_{k}}{\arg \max }\left\{\left[b_{k}(t)\left(m_{S}(i, l)-r\right)\right] W_{x}^{k}(t, x, l, i)\right. \\
& \left.+\frac{1}{2}\left[b_{k}^{2}(t)-2 \kappa_{k} b_{k}(t) b_{m}^{*}(t)\right] \sigma_{S}^{2}(i, l) W_{x x}^{k}(t, x, l, i)\right\},
\end{aligned}
$$

(i) Suppose that

$$
\begin{aligned}
-\frac{\partial W^{k}(t, x, l, i)}{\partial t}-\sup _{\pi_{k} \in \Pi_{k}}\left\{\mathcal{L}^{\pi_{k}} W^{k}(t, x, l, i)\right\} & \geq 0, \\
W^{k}(T, x, l, i) & \geq U_{k}(x), \quad \text { for all }(t, x, l, j) \in \Gamma,
\end{aligned}
$$

then $W^{k} \geq V^{k}$ on $\Gamma$, where $V^{k}$ is defined in (18).

(ii) For $\pi_{k}^{*} \in \Gamma \cap \Pi_{k}$, it follows that

$$
-\frac{\partial W^{k}(t, x, l, i)}{\partial t}-\mathcal{L}^{\pi_{k}^{*}} W^{k}(t, x, l, i)=0
$$

and the SDE

$$
\mathrm{d} \hat{X}_{k}^{\pi_{k}^{*}}(t) \triangleq \mathrm{d} X_{k}^{\pi_{k}^{*}}(t)-\kappa_{k} \mathrm{~d} X_{m}^{\pi_{m}^{*}}(t), \quad m \neq k \in\{1,2\},
$$


admits a unique solution, denoted by $\hat{X}_{k}^{\pi_{k}^{*}}$, given initial condition $\hat{X}_{k}^{\pi_{k}^{*}}(0)=x, \alpha(0)=i$, and $L(0)=l$. Furthermore, the process $\left\{\pi_{k}^{*}(t)\right\}_{t \geq 0}$ lies in $\Pi_{k}$. Then

$$
W^{k}=V^{k}, \quad \text { on } \quad \Gamma,
$$

and $\pi_{k}^{*}$ is an optimal Markovian control.

Proof. (i) As $W^{k} \in \mathcal{C}^{1,2}(\Gamma)$, applying Itô's Lemma to $W^{k}$ gives, for all $(t, x, l, i) \in \Gamma$, $\pi_{k} \in \Pi_{k}, s \geq t$, and any stopping time $\tau$ in $[t, \infty)$,

$$
\begin{aligned}
& W^{k}\left(s \wedge \tau, \hat{X}_{k}^{\pi_{k}}(s \wedge \tau), L(s \wedge \tau), \alpha(s \wedge \tau)\right) \\
= & W^{k}(t, x, l, i)+\int_{t}^{s \wedge \tau}\left\{\frac{\partial W^{k}}{\partial t}\left(u, \hat{X}_{k}^{\pi_{k}}(u), L(u), \alpha(u)\right)+\mathcal{L}^{\pi_{k}} W^{k}\left(u, \hat{X}_{k}^{\pi_{k}}(u), L(u), \alpha(u)\right)\right\} \mathrm{d} u, \\
& +\int_{t}^{s \wedge \tau} D_{x} W^{k}\left(u, \hat{X}_{k}^{\pi_{k}}(u), L(u), \alpha(u)\right) a_{k}(u) \sigma_{k}(\alpha(u), L(u)) \mathrm{d} B_{k}(u) \\
& -\int_{t}^{s \wedge \tau} D_{x} W^{k}\left(u, \hat{X}_{k}^{\pi_{k}}(u), L(u), \alpha(u)\right) \kappa_{k} a_{m}(u) \sigma_{m}(\alpha(u), L(u)) \mathrm{d} B_{m}(u) \\
& +\int_{t}^{s \wedge \tau} D_{x} W^{k}\left(u, \hat{X}_{k}^{\pi_{k}}(u), L(u), \alpha(u)\right)\left(b_{k}(u)-\kappa_{k} b_{m}(u)\right) \sigma_{S}(\alpha(u), L(u)) \mathrm{d} B_{S}(u) \\
& +\int_{t}^{s \wedge \tau} D_{l} W^{k}\left(u, \hat{X}_{k}^{\pi_{k}}(u), L(u), \alpha(u)\right) \sigma_{L}(u, L(u)) \mathrm{d} B_{L}(u) \\
& +\sum_{j \in E} \int_{t}^{s \wedge \tau}\left[W^{k}\left(u, \hat{X}_{k}^{\pi_{k}}(u), L(u), j\right)-W^{k}\left(u, \hat{X}_{k}^{\pi_{k}}(u), L(u), \alpha(u-)\right)\right] \mathrm{d} M(u),
\end{aligned}
$$

where $D_{y}$ denotes the differential operator with respect to $y$.

Suppressing the arguments of the functions, choose

$$
\begin{aligned}
\tau= & \tau_{n} \triangleq \inf \left\{s \geq t ; \int_{t}^{s}\left|D_{x} W^{k}\left(a_{k} \sigma_{k}-\kappa_{k} a_{m} \sigma_{m}\right)\right|^{2} \mathrm{~d} u+\int_{t}^{s}\left|D_{x} W^{k}\left(b_{k}-\kappa_{k} b_{m}\right) \sigma_{S}\right|^{2} \mathrm{~d} u\right. \\
& \left.+\int_{t}^{s}\left|D_{l} W^{k} \sigma_{L}\right|^{2} \mathrm{~d} u+\sum_{j \in E} \int_{t}^{s}\left|\left[W^{k}(., ., ., j)-W^{k}(., ., ., \alpha(u-))\right] 1_{\{\alpha(u-)=i\}} q_{i j}\right|^{2} \mathrm{~d} u \geq n\right\} .
\end{aligned}
$$

Note that $\tau_{n} \uparrow \infty$ as $n \rightarrow \infty$, the stopped processes,

$$
\left\{\begin{array}{l}
\left(\int_{t}^{s \wedge \tau_{n}} D_{x} W^{k}\left(u, \hat{X}_{k}^{\pi_{k}}(u), L(u), \alpha(u)\right) a_{k}(u) \sigma_{k}(\alpha(u), L(u)) \mathrm{d} B_{k}(u)\right)_{\{t \leq s \leq T\}} \\
\left(\int_{t}^{s \wedge \tau_{n}} D_{x} W^{k}\left(u, \hat{X}_{k}^{\pi_{k}}(u), L(u), \alpha(u)\right) \kappa_{k} a_{m}(u) \sigma_{m}(\alpha(u), L(u)) \mathrm{d} B_{m}(u)\right)_{\{t \leq s \leq T\}} \\
\left(\int_{t}^{s \wedge \tau_{n}} D_{x} W^{k}\left(u, \hat{X}_{k}^{\pi_{k}}(u), L(u), \alpha(u)\right)\left(b_{k}(u)-\kappa_{k} b_{m}(u)\right) \sigma_{S}(\alpha(u), L(u)) \mathrm{d} B_{S}(u)\right)_{\{t \leq s \leq T\}} \\
\left(\int_{t}^{s \wedge \tau_{n}} D_{l} W^{k}\left(u, \hat{X}_{k}^{\pi_{k}}(u), L(u), \alpha(u)\right) \sigma_{L}(u, L(u)) \mathrm{d} B_{L}(u)\right)_{\{t \leq s \leq T\}} \\
\left.\left(\sum_{j \in E} \int_{t}^{s \wedge \tau_{n}} W^{k}\left(u, \hat{X}_{k}^{\pi_{k}}(u), L(u), j\right)-W^{k}\left(u, \hat{X}_{k}^{\pi_{k}}(u), L(u), \alpha(u-)\right)\right] \mathrm{d} M(u)\right)_{\{t \leq s \leq T\}}
\end{array}\right.
$$

are true martingales for each $n$. Taking the expectation in (27), we obtain 


$$
\begin{aligned}
& \mathbb{E}\left[W^{k}\left(s \wedge \tau_{n}, \hat{X}_{k}^{\pi_{k}}\left(s \wedge \tau_{n}\right), L\left(s \wedge \tau_{n}\right), \alpha\left(s \wedge \tau_{n}\right)\right)\right] \\
= & W^{k}(t, x, l, i)+\mathbb{E}\left[\int_{t}^{s \wedge \tau_{n}} \frac{\partial W^{k}}{\partial t}\left(u, \hat{X}_{k}^{\pi_{k}}(u), L(u), \alpha(u)\right)+\mathcal{L}^{\pi_{k}} W^{k}\left(u, \hat{X}_{k}^{\pi_{k}}(u), L(u), \alpha(u)\right) \mathrm{d} u\right]
\end{aligned}
$$

As $W^{k}$ satisfies condition (23), we have

$$
\frac{\partial W^{k}}{\partial t}\left(u, \hat{X}_{k}^{\pi_{k}}(u), L(u), \alpha(u)\right)+\mathcal{L}^{\pi_{k}} W^{k}\left(u, \hat{X}_{k}^{\pi_{k}}(u), L(u), \alpha(u)\right) \leq 0, \pi_{k} \in \Pi_{k},
$$

and so

$$
\mathbb{E}\left[W^{k}\left(s \wedge \tau_{n}, \hat{X}_{k}^{\pi_{k}}\left(s \wedge \tau_{n}\right), L\left(s \wedge \tau_{n}\right), \alpha\left(s \wedge \tau_{n}\right)\right)\right] \leq W^{k}(t, x, l, i), \quad \forall \pi_{k} \in \Pi_{k}
$$

As $W^{k}$ satisfies quadratic growth condition, we have, for all $s \in[t, T]$,

$\sup _{j \in E}\left|W^{k}\left(s \wedge \tau_{n}, \hat{X}_{k}^{\pi_{k}}\left(s \wedge \tau_{n}\right), L\left(s \wedge \tau_{n}\right), j\right)\right| \leq C\left(1+\sup _{s \in[t, T]}\left|\hat{X}_{k}^{\pi_{k}}(s)\right|^{2}+\sup _{s \in[t, T]}|L(s)|^{2}\right)$.

Applying the dominated convergence theorem and sending $n$ to infinity in (29), we have, for all $\pi_{k} \in \Pi_{k}$,

$$
\mathbb{E}\left[W^{k}\left(s, \hat{X}_{k}^{\pi_{k}}(s), L(s), \alpha(s)\right)\right] \leq W^{k}(t, x, l, i) .
$$

As $W^{k}$ is continuous with respect to $t$ and $\hat{X}_{k}^{\pi_{k}}(t)$, by sending $s$ to $T$, we can again apply the dominated convergence theorem to obtain

$$
\mathbb{E}\left[U_{k}\left(\hat{X}_{k}^{\pi_{k}}(T)\right)\right] \leq W^{k}(t, x, l, i), \quad \text { for all } \pi_{k} \in \Pi_{k} .
$$

Because the choice of $\pi_{k} \in \Pi_{k}$ is arbitrary, we can deduce that $V^{k} \leq W^{k}$, for all $(t, x, l, i) \in \Gamma$ and attain at $\pi_{k}^{*}$.

(ii) Applying Itô's Lemma to $W^{k}\left(u, \hat{X}_{k}^{\pi_{k}}(u), L(u), \alpha(u)\right)$ between $t \in[0, T)$ and $s \in[t, T)$, we have

$$
\begin{aligned}
& \mathbb{E}\left[W^{k}\left(s, \hat{X}_{k}^{\pi_{k}}(s), L(s), \alpha(s)\right)\right] \\
= & W^{k}(t, x, l, i)+\mathbb{E}\left[\int_{t}^{s} \frac{\partial W^{k}}{\partial t}\left(u, \hat{X}_{k}^{\pi_{k}}(u), L(u), \alpha(u)\right)+\mathcal{L}^{\pi_{k}} W^{k}\left(u, \hat{X}_{k}^{\pi_{k}}(u), L(u), \alpha(u)\right) \mathrm{d} u\right] .
\end{aligned}
$$

By the definition of $\pi_{k}^{*} \in \Pi_{k}$, we have

$$
-\frac{\partial W^{k}(t, x, l, i)}{\partial t}-\mathcal{L}^{\pi_{k}^{*}} W^{k}(t, x, l, i)=0
$$

and so

$$
\mathbb{E}\left[W^{k}\left(s, \hat{X}_{k}^{\pi_{k}^{*}}(s), L(s), \alpha(s)\right)\right]=W^{k}(t, x, l, i) .
$$

Sending $s$ to $T$ and using (30) and the dominated convergence theorem, we have

$$
W^{k}(t, x, l, i)=\mathbb{E}\left[U_{k}\left(\hat{X}_{k}^{\pi_{k}^{*}}(T)\right)\right] .
$$

This implies that $W^{k}(t, x, l, i) \leq V^{k}(t, x, l, i)$ and, together with the result in $(i)$, it follows that $W^{k}=V^{k}$ and $\pi_{k}^{*}$ is an optimal Markovian control. 


\subsection{The proportional reinsurance and investment strategies at equi- librium}

Theorem 4.1 solves the optimal strategy of insurer $k$ in terms of the strategy of insurer $m$, for $k \neq m \in\{1,2\}$. We now complete the formal analysis by providing the sufficient condition that ensures the existence of the equilibrium strategy in Problem 3.1.

Under the assumptions of Theorem 4.1, it readily follows that to solve

$$
V^{k}(t, x, l, i)=\sup _{\pi_{k} \in \Pi_{k}} \mathbb{E}\left[U\left(X_{1}^{\pi_{k}}(T)-\kappa_{k} X_{m}^{\pi_{m}^{*}}(T)\right)\right], \quad \text { for } \quad k \neq m \in\{1,2\},
$$

it suffices to solve $W^{k}$, for $k=1,2$, which satisfies the Hamilton-Jacobi-Bellman (HJB) equation (24).

When $W_{x x}^{k}(t, x, l, j)=0$, for some $(t, x, l, j) \in \Gamma$, where $\Gamma$ is defined in Theorem 4.1, the optimal strategy $\left(a_{k}^{*}, b_{k}^{*}\right)$ of insurer $k \in\{1,2\}$ becomes a corner solution with a value depending on the sign of $W_{x}^{k}(t, x, l, j)$. As the subsequent analysis of this case is relatively straightforward and less interesting, we hereafter consider the case when $W_{x x}^{k}(t, x, l, j) \neq 0$.

Upon suppressing the arguments of the functions, Theorem 4.1 states that the optimal strategy $\left(a_{k}^{*}, b_{k}^{*}\right)$ of insurer $k \in\{1,2\}$, given the strategy of his competitor $m,\left(a_{m}^{*}, b_{m}^{*}\right)$ for $k \neq m \in\{1,2\}$, can be solved as follows:

$$
\left\{\begin{array}{l}
a_{k}^{*}=\arg \max _{a_{k} \in \mathcal{A}_{k}}\left\{\left[\bar{\theta}_{k} a_{k}\right] W_{x}^{k}+\frac{1}{2}\left[a_{k}^{2} \sigma_{k}^{2}-2 \kappa_{k} a_{k} a_{m}^{*} \sigma_{k} \sigma_{m} \rho\right] W_{x x}^{k}\right\} \\
b_{k}^{*}=\arg \max _{b_{k} \in \mathcal{B}_{k}}\left\{\left[b_{k}\left(m_{S}-r\right)\right] W_{x}^{k}+\frac{1}{2}\left[b_{k}^{2}-2 \kappa_{k} b_{k} b_{m}^{*}\right] \sigma_{S}^{2} W_{x x}^{k}\right\} .
\end{array}\right.
$$

Assuming that $W_{x x}^{k}(t, x, l, j) \neq 0$, for $(t, x, l, j) \in \Gamma$, the first order conditions give

$$
\left\{\begin{array}{l}
a_{k}^{*}=\left(\left(\kappa_{k} \rho \frac{\sigma_{m}}{\sigma_{k}}\right) a_{m}^{*}-\frac{\bar{\theta}_{k} W_{x}^{k}}{\sigma_{k}^{2} W_{x x}^{k}} \wedge 1\right)^{+} \\
b_{k}^{*}=\kappa_{k} b_{m}^{*}-\frac{\left(m_{S}-r\right) W_{x}^{k}}{\sigma_{S}^{2} W_{x x}^{k}}
\end{array}\right.
$$

With $a_{k}^{*}, b_{k}^{*}$ in the form of (36), the corresponding HJB equation for insurer $k$ becomes, for $k \neq m \in\{1,2\}$,

$$
\begin{aligned}
0= & W_{t}^{k}+\left[r x+\left(\mu_{k}-\kappa_{k} \mu_{m}\right)-\kappa_{k} \bar{\theta}_{m} a_{m}^{*}-\kappa_{k} b_{m}^{*}\left(m_{S}-r\right)\right] W_{x}^{k} \\
& +\frac{1}{2}\left[\kappa_{k}^{2} a_{m}^{* 2} \sigma_{m}^{2}+\kappa_{k}^{2} b_{m}^{* 2} \sigma_{S}^{2}\right] W_{x x}^{k}+\bar{\theta}_{k} W_{x}^{k}\left[\left(\kappa_{k} \rho \frac{\sigma_{m}}{\sigma_{k}}\right) a_{m}^{*}-\frac{\bar{\theta}_{k} W_{x}^{k}}{\sigma_{k}^{2} W_{x x}^{k}}\right] \\
& +\frac{1}{2}\left[\left(\kappa_{k} \rho \frac{\sigma_{m}}{\sigma_{k}}\right) a_{m}^{*}-\frac{\bar{\theta}_{k} W_{x}^{k}}{\sigma_{k}^{2} W_{x x}^{k}}\right]^{2} \sigma_{k}^{2} W_{x x}^{k}-\kappa_{k} a_{m}^{*} \sigma_{k} \sigma_{m} \rho W_{x x}^{k}\left[\left(\kappa_{k} \rho \frac{\sigma_{m}}{\sigma_{k}}\right) a_{m}^{*}-\frac{\bar{\theta}_{k} W_{x}^{k}}{\sigma_{k}^{2} W_{x x}^{k}}\right] \\
& +\left(m_{S}-r\right) W_{x}^{k}\left[\kappa_{k} b_{m}^{*}-\frac{\left(m_{S}-r\right) W_{x}^{k}}{\sigma_{S}^{2} W_{x x}^{k}}\right]+\frac{1}{2} \sigma_{S}^{2} W_{x x}^{k}\left[\kappa_{k} b_{m}^{*}-\frac{\left(m_{S}-r\right) W_{x}^{k}}{\sigma_{S}^{2} W_{x x}^{k}}\right]^{2} \\
& -\kappa_{k} b_{m}^{*} \sigma_{S}^{2} W_{x x}^{k}\left[\kappa_{k} b_{m}^{*}-\frac{\left(m_{S}-r\right) W_{x}^{k}}{\sigma_{S}^{2} W_{x x}^{k}}\right]+\mu_{L}(t, l) W_{l}^{k}+\frac{1}{2} \sigma_{L}^{2}(t, l) W_{l l}^{k}+\sum_{j \in E} q_{i j} W^{k}(t, x, l, j) \\
= & W_{t}^{k}+\left\{r x+\left(\mu_{k}-\kappa_{k} \mu_{m}\right)-\kappa_{k}\left[\bar{\theta}_{m}-\rho \frac{\sigma_{m}}{\sigma_{k}} \bar{\theta}_{k}\right] a_{m}^{*}\right\} W_{x}^{k}+\frac{1}{2}\left\{\left[\kappa_{k}^{2} \sigma_{m}^{2}-\kappa_{k}^{2} \rho^{2} \sigma_{m}^{2}\right] a_{m}^{* 2}\right\} W_{x x}^{k} \\
& -\frac{1}{2}\left\{\frac{\bar{\theta}_{k}^{2}}{\sigma_{k}^{2}}+\frac{\left(m_{S}-r\right)^{2}}{\sigma_{S}^{2}}\right\} \frac{\left(W_{x}^{k}\right)^{2}}{W_{x x}^{k}}+\mu_{L}(t, l) W_{l}^{k}+\frac{1}{2} \sigma_{L}^{2}(t, l) W_{l l}^{k}+\sum_{j \in E} q_{i j} W^{k}(t, x, l, j), \quad(37)
\end{aligned}
$$


with the terminal conditions

$$
W^{k}(T, x, l, i)=U_{k}(x), \quad \text { for } k=1,2 .
$$

We now have the sufficient condition for the existence of the Nash equilibrium in Problem 3.1 .

Theorem 4.2. Assume that $\kappa_{1} \kappa_{2}<1$. The Nash equilibrium strategy for Problem 3.1 is the solution of the following coupled system of non-linear equations,

$$
\left\{\begin{array}{l}
a_{1}^{*}(t)=\left(\left(\kappa_{1} \rho \frac{\sigma_{2}(i, l)}{\sigma_{1}(i, l)}\right) a_{2}^{*}(t)-\frac{\bar{\theta}_{1}(i, l) W_{x}^{1}(t, x, l, i)}{\sigma_{1}^{2}(i, l) W_{x x}^{1}(t, x, l, i)} \wedge 1\right)^{+} \\
b_{1}^{*}(t)=\kappa_{1} b_{2}^{*}(t)-\frac{\left(m_{S}(i, l)-r\right) W_{x}^{1}(t, x, l, i)}{\sigma_{S}^{2}(i, l) W_{x x}^{1}(t, x, l, i)} \\
a_{2}^{*}(t)=\left(\left(\kappa_{2} \rho \frac{\sigma_{1}(i, l)}{\sigma_{2}(i, l)}\right) a_{1}^{*}(t)-\frac{\bar{\theta}_{2}(i, l) W_{x}^{2}(t, x, l, i)}{\sigma_{2}^{2}(i, l) W_{x x}^{2}(t, x, l, i)} \wedge 1\right)^{+} \\
b_{2}^{*}(t)=\kappa_{2} b_{1}^{*}(t)-\frac{\left(m_{S}(i, l)-r\right) W_{x}^{2}(t, x, l, i)}{\sigma_{S}^{2}(i, l) W_{x x}^{2}(t, x, l, i)}
\end{array}\right.
$$

where $W^{1}$ and $W^{2}$ are the solutions of the following system of coupled partial differential equations (PDEs)

$$
\left\{\begin{aligned}
0=W_{t}^{1}( & t, x, l, i)+\left\{r x+\left(\mu_{1}(i, l)-\kappa_{1} \mu_{2}(i, l)\right)-\kappa_{1}\left[\bar{\theta}_{2}(i, l)-\rho \frac{\sigma_{2}(i, l)}{\sigma_{1}(i, l)} \bar{\theta}_{1}(i, l)\right] a_{2}^{*}(t)\right\} W_{x}^{1}(t, x, l, i) \\
& +\frac{1}{2}\left\{\left[\kappa_{1}^{2} \sigma_{2}^{2}(i, l)-\kappa_{1}^{2} \rho^{2} \sigma_{2}^{2}(i, l)\right] a_{2}^{* 2}(t)\right\} W_{x x}^{1}(t, x, l, i) \\
& -\frac{1}{2}\left\{\frac{\bar{\theta}_{1}^{2}(i, l)}{\sigma_{1}^{2}(i, l)}+\frac{\left(m_{S}(i, l)-r\right)^{2}}{\sigma_{S}^{2}(i, l)}\right\} \frac{\left(W_{x}^{1}(t, x, l, i)\right)^{2}}{W_{x x}^{1}(t, x, l, i)}+\mu_{L}(t, l) W_{l}^{1}(t, x, l, i)+\frac{1}{2} \sigma_{L}^{2}(t, l) W_{l l}^{1}(t, x, l, i) \\
& +\sum_{j \in E} q_{i j} W^{1}(t, x, l, j), \\
0=W_{t}^{2}( & t, x, l, i)+\left\{r x+\left(\mu_{2}(i, l)-\kappa_{2} \mu_{1}(i, l)\right)-\kappa_{2}\left[\bar{\theta}_{1}(i, l)-\rho \frac{\sigma_{1}(i, l)}{\sigma_{2}(i, l)} \bar{\theta}_{2}(i, l)\right] a_{1}^{*}(t)\right\} W_{x}^{2}(t, x, l, i) \\
& +\frac{1}{2}\left\{\left[\kappa_{2}^{2} \sigma_{1}^{2}(i, l)-\kappa_{2}^{2} \rho^{2} \sigma_{1}^{2}(i, l)\right] a_{1}^{* 2}(t)\right\} W_{x x}^{2}(t, x, l, i) \\
& -\frac{1}{2}\left\{\frac{\bar{\theta}_{2}^{2}(i, l)}{\sigma_{2}^{2}(i, l)}+\frac{\left(m_{S}(i, l)-r\right)^{2}}{\sigma_{S}^{2}(i, l)}\right\} \frac{\left(W_{x}^{2}(t, x, l, i)\right)^{2}}{W_{x x}^{2}(t, x, l, i)}+\mu_{L}(t, l) W_{l}^{2}(t, x, l, i)+\frac{1}{2} \sigma_{L}^{2}(t, l) W_{l l}^{2}(t, x, l, i) \\
& +\sum_{j \in E} q_{i j} W^{2}(t, x, l, j),
\end{aligned}\right.
$$

with the terminal condition

$$
\left\{\begin{array}{l}
W^{1}(T, x, l, i)=U_{1}(x) \\
W^{2}(T, x, l, i)=U_{2}(x) .
\end{array}\right.
$$

Remark 4.3. In general, the existence and uniqueness of the solution of the system of coupled PDEs in Theorem 4.2 is very difficult to obtain for any $T>0$. (See, for example, Bensoussan and Frehse (2000) on the connection between the regularities of PDEs and the existence of the Nash equilibrium in the contexts of non-zero sum stochastic differential games.) Nonetheless, for a sufficiently small $T$, the (local) existence and uniqueness of the associated coupled PDEs can easily be ascertained by invoking Cauchy-Kowalevski Theorem.

\section{Example: Nash equilibrium of CARA insurers}

In this section, we show that for the case in which both insurance companies are constant absolute risk aversion (CARA) agents, i.e. each agent has an exponential utility function, we can obtain the explicit value function and the equilibrium strategy for each insurer. 
To this end, we simplify the subsequent analysis by suppressing the role of the market index process $\{L(t)\}_{t \geq 0}$ in this section. This allows us to focus exclusively on the effect of the change in economic states, i.e. $\{\alpha(t)\}_{t \geq 0}$, on the equilibrium strategies and the value functions of the insurance companies.

Denote by $\mathbf{e}_{i}=(0, \cdots, 0,1,0, \cdots, 0)^{\top} \in \mathbb{R}^{d}$ the unit vector with 1 in the $i$-th component and $\mathbf{1}_{d}=(1, \cdots, 1)^{\top} \in \mathbb{R}^{d}$.

The following theorem solves Theorem 4.2 explicitly for the non-zero sum game with two competing CARA insurers.

Theorem 5.1. Assume $\kappa_{1} \kappa_{2}<1$, and that each insurer has an exponential utility function, i.e.

$$
U_{k}(x) \triangleq-\frac{1}{\eta_{k}} \exp \left(-\eta_{k} x\right), \quad \text { for } \quad \eta_{k}>0, \quad k=1,2 .
$$

Then the HJB equation for insurer $k$ in (37) can be solved explicitly with the value function taking the form

$$
W^{k}(t, x, i)=-\frac{1}{\eta_{k}} \exp \left(-\eta_{k} \mathrm{e}^{r(T-t)} x\right) f^{k}(t, i), \quad x \triangleq x_{k}-\kappa_{k} x_{m}, \quad k \neq m \in\{1,2\}
$$

where

$$
f^{k}(t, i)=\mathbf{e}_{i}^{\top} \exp \left(\int_{t}^{T}\left(\left\{g^{k}(s, i)\right\}_{\operatorname{diag}}+\mathbf{Q}\right) \mathrm{d} s\right) \mathbf{1}_{d}
$$

with $\left\{g^{k}(t, i)\right\}_{\text {diag }}$ which is a $d \times d$ diagonal matrix with diagonal elements $g^{k}(t, i), i \in\{1, \ldots, d\}$,

$$
\begin{aligned}
g^{k}(t, i) \triangleq & \eta_{k} \mathrm{e}^{r(T-t)}\left[\left(\mu_{k}(i)-\kappa_{k} \mu_{m}(i)\right)+\left(\bar{\theta}_{k}(i) a_{k}^{*}(t)-\kappa_{k} \bar{\theta}_{m}(i) a_{m}^{*}(t)\right)\right. \\
& \left.+\left(b_{k}^{*}(t)-\kappa_{k} b_{m}^{*}(t)\right)\left(m_{S}(i)-r\right)\right] \\
& -\frac{1}{2} \eta_{k}^{2} \mathrm{e}^{2 r(T-t)}\left[\left(a_{k}^{* 2}(t) \sigma_{k}^{2}(i)-2 \kappa_{k} a_{k}^{*}(t) a_{m}^{*}(t) \sigma_{k}(i) \sigma_{m}(i) \rho+\kappa_{k}^{2} a_{m}^{* 2}(t) \sigma_{m}^{2}(i)\right)\right. \\
& \left.+\sigma_{S}^{2}(i)\left(b_{k}^{* 2}(t)-2 b_{k}^{*}(t) b_{m}^{*}(t) \kappa_{k}+\kappa_{k}^{2} b_{m}^{* 2}(i)\right)\right], \quad m \neq k \in\{1,2\} .
\end{aligned}
$$

Furthermore, the equilibrium strategy $\left(a_{k}^{*}(t), b_{k}^{*}(t)\right) \in \Pi_{k}, k=1,2$, is given as follows. Define

$$
\left\{\begin{array}{l}
\hat{a}_{1}(t) \triangleq \frac{\mathrm{e}^{-r(T-t)}}{1-\kappa_{1} \kappa_{2} \rho^{2}}\left(\frac{\rho \kappa_{1} \bar{\theta}_{2}(i)}{\sigma_{1}(i) \sigma_{2}(i) \eta_{2}}+\frac{\bar{\theta}_{1}(i)}{\sigma_{1}^{2}(i) \eta_{1}}\right), \\
\hat{a}_{2}(t) \triangleq \frac{\mathrm{e}^{-r(T-t)}}{1-\kappa_{1} \kappa_{2} \rho^{2}}\left(\frac{\rho \kappa_{2} \bar{\theta}_{1}(i)}{\sigma_{1}(i) \sigma_{2}(i) \eta_{1}}+\frac{\bar{\theta}_{2}(i)}{\sigma_{2}^{2}(i) \eta_{2}}\right) .
\end{array}\right.
$$

For $a_{k}^{*}(t), k=1,2$, we have the following cases:

1. If $\hat{a}_{1}(t) \geq 0$ and $\hat{a}_{2}(t) \geq 0$, then

(a) If $\hat{a}_{1}(t) \leq 1$ and $\hat{a}_{2}(t) \leq 1$, then $\left(a_{1}^{*}(t), a_{2}^{*}(t)\right)=\left(\hat{a}_{1}(t), \hat{a}_{2}(t)\right)$.

(b) If $\hat{a}_{1}(t) \leq 1$ and $\hat{a}_{2}(t)>1$, then $\left(a_{1}^{*}(t), a_{2}^{*}(t)\right)=\left(\kappa_{1} \rho \frac{\sigma_{2}(i)}{\sigma_{1}(i)}+\frac{\mathrm{e}^{-r(T-t)} \bar{\theta}_{1}(i)}{\sigma_{1}^{2}(i) \eta_{1}}, 1\right)$. 
(c) If $\hat{a}_{1}(t)>1$ and $\hat{a}_{2}(t) \leq 1$, then $\left(a_{1}^{*}(t), a_{2}^{*}(t)\right)=\left(1, \kappa_{2} \rho \frac{\sigma_{1}(i)}{\sigma_{2}(i)}+\frac{\mathrm{e}^{-r(T-t)} \bar{\theta}_{2}(i)}{\sigma_{2}^{2}(i) \eta_{2}}\right)$.

(d) If $\hat{a}_{1}(t)>1$ and $\hat{a}_{2}(t)>1$, then $\left(a_{1}^{*}(t), a_{2}^{*}(t)\right)=(1,1)$.

2. Otherwise, the following statements are true.

Define

$$
\gamma \triangleq \frac{\bar{\theta}_{1}(i) \sigma_{2}(i) \eta_{2}}{\bar{\theta}_{2}(i) \sigma_{1}(i) \eta_{1}}
$$

(a) If $\kappa_{2} \gamma \geq-\frac{1}{\rho}$ and $\frac{1}{\kappa_{1}} \gamma \geq-\rho$, then

$$
\left(a_{1}^{*}(t), a_{2}^{*}(t)\right)=\left(\left(\frac{\mathrm{e}^{-r(T-t)} \bar{\theta}_{1}(i)}{\sigma_{1}^{2}(i) \eta_{1}}\right) \wedge 1,\left(\kappa_{2} \rho \frac{\sigma_{1}(i)}{\sigma_{2}(i)}+\frac{\mathrm{e}^{-r(T-t)} \bar{\theta}_{2}(i)}{\sigma_{2}^{2}(i) \eta_{2}}\right)^{+}\right) .
$$

(b) If $\kappa_{2} \gamma<-\frac{1}{\rho}$ and $\frac{1}{\kappa_{1}} \gamma<-\rho$, then

$$
\left(a_{1}^{*}(t), a_{2}^{*}(t)\right)=\left(\left(\kappa_{1} \rho \frac{\sigma_{2}(i)}{\sigma_{1}(i)}+\frac{\mathrm{e}^{-r(T-t)} \bar{\theta}_{1}(i)}{\sigma_{1}^{2}(i) \eta_{1}}\right)^{+},\left(\frac{\mathrm{e}^{-r(T-t)} \bar{\theta}_{2}(i)}{\sigma_{2}^{2}(i) \eta_{2}}\right) \wedge 1\right) .
$$

For $b_{k}^{*}(t), k=1,2$, we have

$$
\left\{\begin{array}{l}
b_{1}^{*}(t)=\frac{\mathrm{e}^{-r(T-t)}\left(m_{S}(i)-r\right)}{\left(1-\kappa_{1} \kappa_{2}\right) \sigma_{S}^{2}(i)}\left(\frac{1}{\eta_{1}}+\kappa_{1} \frac{1}{\eta_{2}}\right) \\
b_{2}^{*}(t)=\frac{\mathrm{e}^{-r(T-t)}\left(m_{S}(i)-r\right)}{\left(1-\kappa_{1} \kappa_{2}\right) \sigma_{S}^{2}(i)}\left(\frac{1}{\eta_{2}}+\kappa_{2} \frac{1}{\eta_{1}}\right) .
\end{array}\right.
$$

Remark 5.2. Theorem 5.1 can also be enriched to include the market index process $\{L(t)\}_{t \geq 0}$, albeit the equilibrium value functions of the insurers would then be in terms of both $\alpha_{t}$ and $L_{t}$. One key observation is that the equilibrium strategies of the insurers remain unchanged in the presence of the market index process $\{L(t)\}_{t \geq 0}$. This claim can easily be verified by recalling the assumption that the Brownian motion $\left\{B_{L}(t)\right\}_{t \geq 0}$ in the market index process $\{L(t)\}_{t \geq 0}$ is independent of $\left\{B_{k}(t)\right\}_{t \geq 0}, k=1,2$, and $\left\{B_{S}(t)\right\}_{t \geq 0}$, the Brownian motions of the insurers' surplus processes.

Remark 5.3. As discussed in the introduction, the problem of minimizing the probability of ruin has a long history in the insurance literature. Ferguson (1965) conjectured the equivalence of maximizing the expected exponential utility on the terminal wealth and minimizing the probability of ruin, which was confirmed by Browne (1995) in the single-risky-asset case. Together with its analytical tractability, this confirmation has attracted many researchers to investigate the optimal proportional reinsurance/investment/consumption strategy within the exponential utility framework under different asset and claim dynamics. In this respect, it would be interesting to revisit this conjecture in the context of competition considered in this paper.

Proof. (Proof of Theorem 5.1)

We begin with an Ansatz, i.e.

$$
W^{k}(t, x, i)=-\frac{1}{\eta_{k}} \exp \left(-\eta_{k} \mathrm{e}^{r(T-t)} x\right) f^{k}(t, i), \quad \text { for } \quad k=1,2,
$$


where $f^{k}:[0, T] \times E \rightarrow \mathbb{R}_{+}$is to be determined.

Let $h^{k}(t, x)=\frac{1}{\eta_{k}} \exp \left(-\eta_{k} \mathrm{e}^{r(T-t)} x\right)$, where $x=x_{k}-\kappa_{k} x_{m}$, for $k \neq m \in\{1,2\}$, then we have

$$
\begin{aligned}
W_{t}^{k}(t, x, i) & =-\eta_{k} r \mathrm{e}^{r(T-t)} x h^{k}(t, x) f^{k}(t, i)-h^{k}(t, x) f_{t}^{k}(t, i), \\
W_{x}^{k}(t, x, i) & =\eta_{k} \mathrm{e}^{r(T-t)} h^{k}(t, x) f^{k}(t, i), \\
W_{x x}^{k}(t, x, i) & =-\eta_{k}^{2} \mathrm{e}^{2 r(T-t)} h^{k}(t, x) f^{k}(t, i) .
\end{aligned}
$$

The corresponding HJB equation in (37) becomes

$$
\begin{aligned}
0= & -\eta_{k} r \mathrm{e}^{r(T-t)}\left(x_{k}-\kappa_{k} x_{m}\right) h^{k}(t, x) f^{k}(t, i)-f_{t}^{k}(t, i) h^{k}(t, x) \\
& +\left[\left(\mu_{k}+r x_{k}\right)-\kappa_{k}\left(\mu_{m}(i)+r x_{m}\right)-\kappa_{k} \bar{\theta}_{m}(i) a_{m}^{*}(t)-\kappa_{k} b_{m}^{*}\left(m_{S}(i)-r\right)\right] \eta_{k} \mathrm{e}^{r(T-t)} h^{k}(t, x) f^{k}(t, i) \\
& -\frac{1}{2}\left[\kappa_{k}^{2} a_{m}^{* 2}(t) \sigma_{m}^{2}(i)+\kappa_{k}^{2} b_{m}^{* 2}(t) \sigma_{S}^{2}(i)\right] \eta_{k}^{2} \mathrm{e}^{2 r(T-t)} h^{k}(t, x) f^{k}(t, i) \\
& +\inf _{a_{k} \in \mathcal{A}_{k}}\left\{\bar{\theta}_{k}(i) a_{k}(t) \eta_{k} \mathrm{e}^{r(T-t)} h^{k}(t, x) f^{k}(t, i)\right. \\
& \left.-\frac{1}{2}\left[a_{k}^{2}(t) \sigma_{k}^{2}(i)-2 a_{k}(t) a_{m}^{*}(t) \kappa_{k} \sigma_{k}(i) \sigma_{m}(i) \rho\right] \eta_{k}^{2} \mathrm{e}^{2 r(T-t)} h^{k}(t, x) f^{k}(t, j)\right\} \\
& +\inf _{b_{k} \in \mathcal{B}_{k}}\left\{b_{k}(t)\left(m_{S}(i)-r\right) \eta_{k} \mathrm{e}^{r(T-t)} h^{k}(t, x) f^{k}(t, i)\right. \\
& \left.-\frac{1}{2}\left[b_{k}^{2}(t)-2 b_{k}(t) b_{m}^{*}(t) \kappa_{k}\right] \sigma_{S}^{2}(i) \eta_{k}^{2} \mathrm{e}^{2 r(T-t)} h^{k}(t, x) f^{k}(t, i)\right\}-\sum_{j \in E} q_{i j} h^{k}(t, x) f^{k}(t, i),
\end{aligned}
$$

and after rearranging the terms, we have

$$
\left\{\begin{aligned}
f_{t}^{k}(t, i)= & \left\{\eta_{k} \mathrm{e}^{r(T-t)}\left[\left(\mu_{k}(i)-\kappa_{1} \mu_{m}(i)\right)-\kappa_{1} \bar{\theta}_{m}(i) a_{m}^{*}(t)-\kappa_{1} b_{m}^{*}(t)\left(m_{S}(i)-r\right)\right]\right. \\
& -\frac{1}{2} \eta_{k}^{2} \mathrm{e}^{2 r(T-t)} \kappa_{k}^{2}\left[a_{m}^{* 2}(t) \sigma_{m}^{2}(i)+b_{m}^{* 2}(t) \sigma_{S}^{2}(i)\right] \\
& +\inf _{a_{k} \in \mathcal{A}_{k}}\left[\eta_{1} \mathrm{e}^{r(T-t)} \bar{\theta}_{k}(i) a_{k}(t)-\frac{1}{2} \eta_{k}^{2} \mathrm{e}^{2 r(T-t)}\left(a_{k}^{2}(t) \sigma_{k}^{2}(i)-2 a_{k}(t) a_{m}^{*}(t) \kappa_{k} \sigma_{k}(i) \sigma_{m}(i) \rho\right)\right] \\
& \left.+\inf _{b_{k} \in \mathcal{B}_{k}}\left[\eta_{k} \mathrm{e}^{r(T-t)} b_{k}(t)\left(m_{S}(i)-r\right)-\frac{1}{2} \eta_{k}^{2} \sigma_{S}^{2}(i)\left(b_{k}^{2}(t)-2 b_{k}(t) b_{m}^{*}(t) \kappa_{1}\right)\right]\right\} f^{k}(t, i) \\
& -\sum_{j \in E} q_{i j} f^{k}(t, j) \\
f^{k}(T, i)= & 1, \quad \text { for all } i \in\{1, \ldots, d\} .
\end{aligned}\right.
$$

With (50) and (51) in explicit forms, we now derive the corresponding $a_{k}^{*}(t)$ and $b_{k}^{*}(t)$, for $k=1,2$. 
Observe that the equations

$$
\begin{aligned}
& a_{1}^{*}(t)=\underset{a_{1} \in \mathcal{A}_{1}}{\arg \min }\{\left.\bar{\theta}_{1}(i) a_{1}(t)\right] \eta_{1} \mathrm{e}^{r(T-t)} h^{1}(t, x) f^{1}(t, i) \\
&\left.-\frac{1}{2}\left[a_{1}^{2} \sigma_{1}^{2}(i)-2 a_{1}(t) a_{2}^{*}(t) \kappa_{1} \sigma_{1}(i) \sigma_{2}(i) \rho\right] \eta_{1}^{2} \mathrm{e}^{2 r(T-t)} h^{1}(t, x) f^{1}(t, i)\right\}, \\
& a_{2}^{*}(t)=\underset{a_{2} \in \mathcal{A}_{2}}{\arg \min }\left\{\left[\bar{\theta}_{2}(i) a_{2}(t)\right] \eta_{2} \mathrm{e}^{r(T-t)} h^{2}(t, x) f^{2}(t, i)\right. \\
&\left.\quad-\frac{1}{2}\left[a_{2}^{2}(t) \sigma_{2}^{2}(i)-2 a_{2}(t) a_{1}^{*}(t) \kappa_{2} \sigma_{1}(i) \sigma_{2}(i) \rho\right] \eta_{2}^{2} \mathrm{e}^{2 r(T-t)} h^{2}(t, x) f^{2}(t, i)\right\},
\end{aligned}
$$

are, respectively, equivalent to

$$
\left\{\begin{array}{l}
a_{1}^{*}(t)=\left(\kappa_{1} \rho \frac{\sigma_{2}(i)}{\sigma_{1}(i)}\right) a_{2}^{*}(t)+\frac{\mathrm{e}^{-r(T-t)} \bar{\theta}_{1}(i)}{\eta_{1} \sigma_{1}^{2}(i)} \\
a_{2}^{*}(t)=\left(\kappa_{2} \rho \frac{\sigma_{1}(i)}{\sigma_{2}(i)}\right) a_{1}^{*}(t)+\frac{\mathrm{e}^{-r(T-t)} \bar{\theta}_{2}(i)}{\eta_{2} \sigma_{2}^{2}(i)}
\end{array}\right.
$$

For notational convenience, we suppress the argument of the functions.

First, let us consider the case when $\rho \in[0,1]$. As the case when $\rho=0$ is straightforward, we focus on the case when $\rho \in(0,1]$.

Define lines $l_{1}$ and $l_{2}$ as follows

$$
\begin{aligned}
& l_{1}: a_{1}=\left(\kappa_{1} \rho \frac{\sigma_{2}}{\sigma_{1}}\right) a_{2}+\frac{\mathrm{e}^{-r(T-t)} \bar{\theta}_{1}}{\eta_{1} \sigma_{1}^{2}}, \\
& l_{2}: a_{2}=\left(\kappa_{2} \rho \frac{\sigma_{1}}{\sigma_{2}}\right) a_{1}+\frac{\mathrm{e}^{-r(T-t)} \bar{\theta}_{2}}{\eta_{2} \sigma_{2}^{2}} .
\end{aligned}
$$

As $\kappa_{1}, \kappa_{2}, \sigma_{1}, \sigma_{2}, \rho>0$, the lines $l_{1}$ and $l_{2}$ have positive slopes and intersect with each other at $\left(\hat{a}_{1}, \hat{a}_{2}\right)$, defined in (45), which lies in the first quadrant of the $\left(a_{1}, a_{2}\right)$-plane. From this observation, it becomes clear that if $\hat{a}_{k} \leq 1, k=1,2, a_{1}^{*}=\hat{a}_{1}$ and $a_{2}^{*}=\hat{a}_{2}$, thus proving Case $1(a)$. Furthermore, for $\hat{a}_{k}>1, k=1,2$, we have $a_{1}^{*}=1$ and $a_{2}^{*}=1$, thus proving Case $1(d)$.

Consider Case $1(c)$, when $\hat{a}_{1} \leq 1$ and $\hat{a}_{2}>1$. The graph of

$$
a_{2}=\left(\kappa_{2} \rho \frac{\sigma_{1}}{\sigma_{2}} a_{1}+\frac{\mathrm{e}^{-r(T-t)} \bar{\theta}_{2}}{\eta_{2} \sigma_{2}^{2}}\right) \wedge 1
$$

intersects $l_{1}$ at

$$
\left(\left(\kappa_{1} \rho \frac{\sigma_{2}}{\sigma_{1}}\right)+\frac{\mathrm{e}^{-r(T-t)} \bar{\theta}_{1}}{\eta_{1} \sigma_{1}^{2}}, 1\right) .
$$

Case $1(b)$, i.e. when $\hat{a}_{1}>1$ and $\hat{a}_{2} \leq 1$, can be proven analogously.

Now consider the case when $\rho \in[-1,0)$. 
Let $h_{11}$ and $h_{21}$ denote the $a_{1}$-intercepts of lines $l_{1}$ and $l_{2}$, respectively. Similarly, let $h_{12}$ and $h_{22}$ denote the $a_{2}$-intercepts of lines $l_{1}$ and $l_{2}$, respectively. Then, it follows that

$$
\begin{array}{lll}
h_{11} \triangleq \frac{\mathrm{e}^{-r(T-t)} \bar{\theta}_{1}}{\eta_{1} \sigma_{1}^{2}}, & h_{21} \triangleq-\frac{\mathrm{e}^{-r(T-t)} \bar{\theta}_{2}}{\eta_{2} \kappa_{2} \rho \sigma_{1} \sigma_{2}}, \\
h_{12} \triangleq-\frac{\mathrm{e}^{-r(T-t)} \bar{\theta}_{1}}{\eta_{1} \kappa_{1} \rho \sigma_{1} \sigma_{2}}, & h_{22} \triangleq \frac{\mathrm{e}^{-r(T-t)} \bar{\theta}_{2}}{\eta_{2} \sigma_{2}^{2}} .
\end{array}
$$

This readily implies that when $\rho \in[-1,0), h_{11}, h_{21}, h_{12}, h_{22} \geq 0$.

Let $\gamma$ be defined in (46). We then have four cases:

(i) $h_{11} \geq h_{21}$ and $h_{12} \geq h_{22}$.

In this case, we have $\kappa_{2} \gamma \geq-\frac{1}{\rho}$ and $\frac{1}{\kappa_{1}} \gamma \geq-\rho$. If $h_{11} \leq 1$, the graph of

$$
a_{2}=\left(\kappa_{2} \rho \frac{\sigma_{1}}{\sigma_{2}} a_{1}+\frac{\mathrm{e}^{-r(T-t)} \bar{\theta}_{2}}{\eta_{2} \sigma_{2}^{2}}\right) \vee 0
$$

will intersect $l_{1}$ at $\left(h_{11}, 0\right)$. However, if $h_{11}>1$, then the graph of

$$
a_{1}=\left(\kappa_{1} \rho \frac{\sigma_{2}}{\sigma_{1}} a_{2}+\frac{\mathrm{e}^{-r(T-t)} \bar{\theta}_{1}}{\eta_{1} \sigma_{1}^{2}}\right) \wedge 1
$$

will intersect $l_{2}$ at

$$
\left(1,\left(\kappa_{2} \rho \frac{\sigma_{1}}{\sigma_{2}}+\frac{\mathrm{e}^{-r(T-t)} \bar{\theta}_{2}}{\eta_{2} \sigma_{2}^{2}}\right)^{+}\right)
$$

(ii) $h_{11} \geq h_{21}$ and $h_{12}<h_{22}$

In this case, as $\kappa_{1} \kappa_{2}<1$ and $\gamma \geq 0$, we have

$$
-\rho \geq \frac{1}{\kappa_{1}} \gamma>\kappa_{2} \gamma>-\frac{1}{\rho}
$$

which is a contradiction because $\rho \in[-1,0)$.

(iii) $h_{11}<h_{21}$ and $h_{12} \geq h_{22}$

In this case, $h_{11}<h_{21}$ is equivalent to $\hat{a}_{2}>0$, while $h_{12} \geq h_{22}$ is equivalent to $\hat{a}_{1}>0$. We then proceed as in the case when $\rho \in[0,1]$ to compute $\left(a_{1}^{*}, a_{2}^{*}\right)$.

(iv) $h_{11}<h_{21}$ and $h_{12}<h_{22}$

In this case, we have $\kappa_{2} \gamma<-\frac{1}{\rho}$ and $\frac{1}{\kappa_{1}} \gamma<-\rho$. We then proceed as in Case $(i)$ to find $\left(a_{1}^{*}, a_{2}^{*}\right)$. 
To find $b_{k}^{*}, k=1,2$, solving

$$
\left\{\begin{array}{r}
b_{1}^{*}=\arg \min _{b_{1} \in \mathcal{B}_{1}}\left\{\left[b_{1}\left(m_{S}-r\right)\right] \eta_{1} \mathrm{e}^{r(T-t)} h^{1}(t, x) f^{1}(t, i)\right. \\
\left.-\frac{1}{2}\left[b_{1}^{2}-2 \kappa_{1} b_{1} b_{2}^{*}\right] \sigma_{S}^{2} \eta_{1}^{2} \mathrm{e}^{2 r(T-t)} h^{1}(t, x) f^{1}(t, i)\right\} \\
b_{2}^{*}=\arg \min _{b_{2} \in \mathcal{B}_{2}}\left\{\left[b_{2}\left(m_{S}-r\right)\right] \eta_{2} \mathrm{e}^{r(T-t)} h^{2}(t, x) f^{2}(t, i)\right. \\
\left.-\frac{1}{2}\left[b_{2}^{2}-2 \kappa_{2} b_{2} b_{1}^{*}\right] \sigma_{S}^{2} \eta_{2}^{2} \mathrm{e}^{2 r(T-t)} h^{2}(t, x) f^{2}(t, i)\right\}
\end{array}\right.
$$

simultaneously gives

$$
b_{1}^{*}=\frac{\mathrm{e}^{-r(T-t)}\left(m_{S}-r\right)}{\left(1-\kappa_{1} \kappa_{2}\right) \sigma_{S}^{2}}\left(\frac{1}{\eta_{1}}+\kappa_{1} \frac{1}{\eta_{2}}\right),
$$

and

$$
b_{2}^{*}=\frac{\mathrm{e}^{-r(T-t)}\left(m_{S}-r\right)}{\left(1-\kappa_{1} \kappa_{2}\right) \sigma_{S}^{2}}\left(\frac{1}{\eta_{2}}+\kappa_{2} \frac{1}{\eta_{1}}\right) .
$$

It is now clear that the equilibrium strategy $\left(\left(a_{1}^{*}, b_{1}^{*}\right),\left(a_{2}^{*}, b_{2}^{*}\right)\right) \in \Pi_{1} \times \Pi_{2}$ depends only on the Markov chain $\{\alpha(t)\}_{t \geq 0}$. The resulting HJB equation for insurer $k$ becomes the following matrix ordinary differential equation (ODE),

$$
\left\{\begin{aligned}
f_{t}^{k}(t, i)= & \left\{\eta _ { k } \mathrm { e } ^ { r ( T - t ) } \left[\left(\mu_{k}(i)-\kappa_{k} \mu_{m}(i)\right)+\left(\bar{\theta}_{k}(i) a_{k}^{*}(t)-\kappa_{k} \bar{\theta}_{m}(i) a_{m}^{*}(t)\right)\right.\right. \\
& \left.+\left(b_{k}^{*}(t)-\kappa_{k} b_{m}^{*}(t)\right)\left(m_{S}(i)-r\right)\right] \\
& -\frac{1}{2} \eta_{k}^{2} \mathrm{e}^{2 r(T-t)}\left[\left(a_{k}^{* 2}(t) \sigma_{k}^{2}(i)-2 \kappa_{k} a_{k}^{*}(t) a_{m}^{*}(t) \sigma_{k}(i) \sigma_{m}(i) \rho+\kappa_{k}^{2} a_{m}^{* 2}(t) \sigma_{m}^{2}(i)\right)\right. \\
& \left.\left.+\sigma_{S}^{2}(i)\left(b_{k}^{* 2}(t)-2 b_{k}^{*}(t) b_{m}^{*}(t) \kappa_{k}+\kappa_{k}^{2} b_{m}^{* 2}(t)\right)\right]\right\} f^{k}(t, i) \\
& -\sum_{j \in E} q_{i j} f^{k}(t, j) \\
f^{k}(T, i)= & 1, \quad \text { for all } i \in\{1, \ldots, d\} .
\end{aligned}\right.
$$

Solving the system of ODEs in (59), we have

$$
f^{k}(t, i)=\mathbf{e}_{i}^{\top} \exp \left(\int_{t}^{T}\left(\left\{g^{k}(s, i)\right\}_{\text {diag }}+\mathbf{Q}\right) \mathrm{d} s\right) \mathbf{1}_{d}
$$

where $g^{k}(t, i)$ takes the form in $(44)$.

Theorem 5.1 explicitly provides the strategy and value function of each insurer at equilibrium under the case of the exponential utility. Observe that the equilibrium reinsurance strategy $\left(a_{1}^{*}(t), a_{2}^{*}(t)\right)$ is presented in a case-by-case manner, to ensure that the equilibrium strategy on 
the reinsurance protection is indeed a proportional reinsurance protection, i.e. $\left(a_{1}^{*}(t), a_{2}^{*}(t)\right) \in$ $[0,1] \times[0,1]$

As $\eta_{k}>0$, for $k=1,2$, and $\kappa_{1} \kappa_{2}<1$, the equilibrium investment strategy is positive if the rate of return of the risky asset $S(t)$ is greater than the risk-free interest rate $r$, i.e. $b_{k}^{*}(t) \geq 0$ if $m_{S} \geq r$. In fact, the equilibrium investment strategy $\left(b_{1}^{*}(t), b_{2}^{*}(t)\right)$ at time $t$ in (47) indicates that it is directly proportional to the Sharpe ratio of the risky asset, which is denoted hereafter as $\mathcal{S}(t) \triangleq \frac{m_{S}(\alpha(t))-r}{\sigma_{S}(\alpha(t))}$, at time $t$. That is,

$$
b_{k}^{*}(t)=\frac{\mathrm{e}^{-r(T-t)}}{\left(1-\kappa_{1} \kappa_{2}\right) \sigma_{S}(i)}\left(\frac{1}{\eta_{k}}+\kappa_{k} \frac{1}{\eta_{m}}\right) \mathcal{S}(t), \quad \text { for } \quad k \neq m \in\{1,2\}
$$

This implies that as long as the Sharpe ratio $\mathcal{S}(t)$ is positive, the insurer will hold the risky asset $S$; the opposite would occur if the risky asset offered a smaller return than that of the risk-free bond. More importantly, the notion of competition is reflected by the sensitivity parameter $\kappa_{k}$. As increasing $\kappa_{k}$ implies that insurer $k$ has become more concerned with the performance of his competitor $m \neq k$, a rise in $\kappa_{k}$ will result in an increase in the holding of the risky asset $S$, ceteris paribus. We provide more detailed comparative-static analyses on the equilibrium strategy $\left(a_{k}^{*}(t), b_{k}^{*}(t)\right)$ in Section 5.1.

In the absence of competition, i.e. $\kappa_{1}=\kappa_{2}=0$, the equilibrium strategy $\left(a_{k}^{*}(t), b_{k}^{*}(t)\right)$, for $k=1,2$, of insurer $k$ would simply be the optimal proportional reinsurance and investment strategy arising from the classical (single-agent) utility maximization problem; see, for example, Liu and Ma (2009).

Three important features of the equilibrium strategy $\left(a_{k}^{*}(t), b_{k}^{*}(t)\right)$, for $k=1,2$, are inherited from the single-agent, utility-maximization framework. The first feature is that although the equilibrium strategy $\left(a_{k}^{*}(t), b_{k}^{*}(t)\right)$, for $k=1,2$, is a function of the current state of the Markov chain $\{\alpha(t)\}_{t \geq 0}$, it does not depend on its intensity $\left(q_{i j}\right)_{i, j \in E}=\mathbf{Q}$. However, the intensity of the regime-switch $\mathbf{Q}$ is reflected in the equilibrium value function $W^{k}$, for $k=1,2$.

Second, the equilibrium strategy $\left(a_{k}^{*}(t), b_{k}^{*}(t)\right)$, for $k=1,2$, of insurer $k$ is independent of his current level of wealth. It is intrinsic to the nature of an exponential utility agent that his optimal decision is independent of the current level of his wealth. The direct consequence is that the corresponding coupled PDEs in (40) become a system of matrix ODEs, in which analytical solutions of the insurance companies' value functions exist.

Finally, the equilibrium proportional reinsurance strategy $a_{k}^{*}$ and the equilibrium investment strategy $b_{k}^{*}$ are separated in the sense that given the risk-aversion parameter $\eta_{k}$, a change in the purchase of the proportional reinsurance will not affect the investment in the risky asset $S$, and vice versa. This means that the CARA-insurer $k$ can set up two separate funds based on his risk-aversion level: one for the purchase of reinsurance protection and the other for the investment in the risky asset. For related works on the three aforementioned features in a single-agent optimal portfolio selection problem with regime-switching under a class of utility functions, see, for example, Bäuerle and Rieder (2004), Sotomayor and Cadenillas (2009), Çanakoğlu and Özekici (2010), Elliott and Siu (2011b), Busch et al. (2013), and the references therein. 


\subsection{Numerical illustrations}

To better understand the effects of the model parameters on the equilibrium strategy $a_{k}^{*}(t)$ and $b_{k}^{*}(t)$, for $k=1,2$, we now turn to study some numerical examples. Unless otherwise stated, we assume the model parameters under the current economic regime $(t=0)$ are as shown in Table 1.

\begin{tabular}{|c|c|c|c|c|c|}
\hline & \multicolumn{4}{|c|}{ Base parameters } & \\
\hline & $\sigma_{S}$ & $r$ & $m_{S}$ & $T$ & \\
\hline & 0.4 & $=0.05$ & 0.2 & 4 & \\
\hline \multicolumn{6}{|c|}{ Insurer 1} \\
\hline$\theta_{1}$ & $\lambda_{1}$ & $\mathbb{E}\left[\xi_{1}\right]$ & $\mathbb{E}\left[\xi_{1}^{2}\right]$ & $\eta_{1}$ & $\kappa_{1}$ \\
\hline 7 & 0.8 & 2.5 & 80 & 0.1 & 0.7 \\
\hline \multicolumn{6}{|c|}{ Insurer 2} \\
\hline$\theta_{2}$ & $\lambda_{2}$ & $\mathbb{E}\left[\xi_{2}\right]$ & $\mathbb{E}\left[\xi_{2}^{2}\right]$ & $\eta_{2}$ & $\kappa_{2}$ \\
\hline 4 & 0.5 & 2 & 50 & 0.3 & 0.5 \\
\hline
\end{tabular}

Table 1: Model parameters

Figure 1 provides a graphical illustration of Theorem 5.1 of $a_{k}^{*}(0)$, for $k=1,2$. The top row of Figure 1 shows the effect of the risk-aversion parameter of insurer $k\left(\eta_{k}\right)$ on his proportional reinsurance strategy $a_{k}^{*}(0)$ at equilibrium when the insurance companies are positively correlated $(\rho=0.5)$, while the bottom row displays the case of a negative correlation $(\rho=-0.5)$. Observe first that $a_{k}^{*}(0)$ is a decreasing function of the risk-aversion parameter $\eta_{k}$. This is in agreement with the optimal reinsurance strategy without competition in the existing literature. Because $a_{k}^{*}(0)$ denotes the proportion that insurer $k$ retains upon purchasing a proportional reinsurance protection, the higher the risk-aversion parameter $\eta_{k}$, the greater the risk that insurer $k$ transfers to the reinsurance company, i.e. higher $\left(1-a_{k}^{*}(0)\right) \%$, which in turn implies lower $a_{k}^{*}(0) \%$. Additionally, the conditions on $a_{k}^{*}(0)$ in Theorem 5.1 ensure that $a_{k}^{*}(0) \in[0,1]$, which is particularly apparent in the second column of Figure 1, where the effect of $\eta_{2}$ on $a_{2}^{*}(0)$ is displayed for the case when $\rho=0.5$ and $\rho=-0.5$.

To illustrate the effect of competition, Figure 1 also displays the effect of insurer $k$ 's riskaversion parameter $\eta_{k}$ on his proportional reinsurance strategy $a_{k}^{*}(0)$, for $k=1,2$, when $\kappa_{k}=0, \kappa_{k}=0.3$, and $\kappa_{k}=0.7$. As parameter $\kappa_{k}$ captures the degree of dependence on the terminal wealth of insurer $k$ 's competitor (see Problem 3.1), a higher $\kappa_{k}$ results in insurer $k$ becoming more concerned with his performance compared with that of his competitor in the terminal period $T$. While purchasing proportional reinsurance can reduce the risk borne by insurer $k$, it is nonetheless costly because insurer $k$ needs to pay $\left(1-a_{k}^{*}(0)\right) \theta_{k}$ to the reinsurance company for the reinsurance protection (see (6)), which decreases his terminal wealth value relative to that of his competitor, $X_{k}(T)-\kappa_{k} X_{m}(T)$, for $k \neq m \in\{1,2\}$. In the case when the correlation between the insurance companies is positive, i.e. $\rho=0.5$, insurer $k$ tends to decrease his expenditure on the reinsurance company, i.e. smaller $\left(1-a_{k}^{*}(0)\right) \theta_{k}$, which increases the dependence parameter $\kappa_{k}$, which in turn implies increasing $a_{k}^{*}(0)$. The top row of Figure 1 summarizes this observation. The opposite results occur when the insurance companies are negatively correlated, i.e. $\rho=-0.5$. In this case, increasing the dependence parameter $\kappa_{k}$ would result in decreasing $a_{k}^{*}(0)$, as shown in the bottom row of Figure 1. 

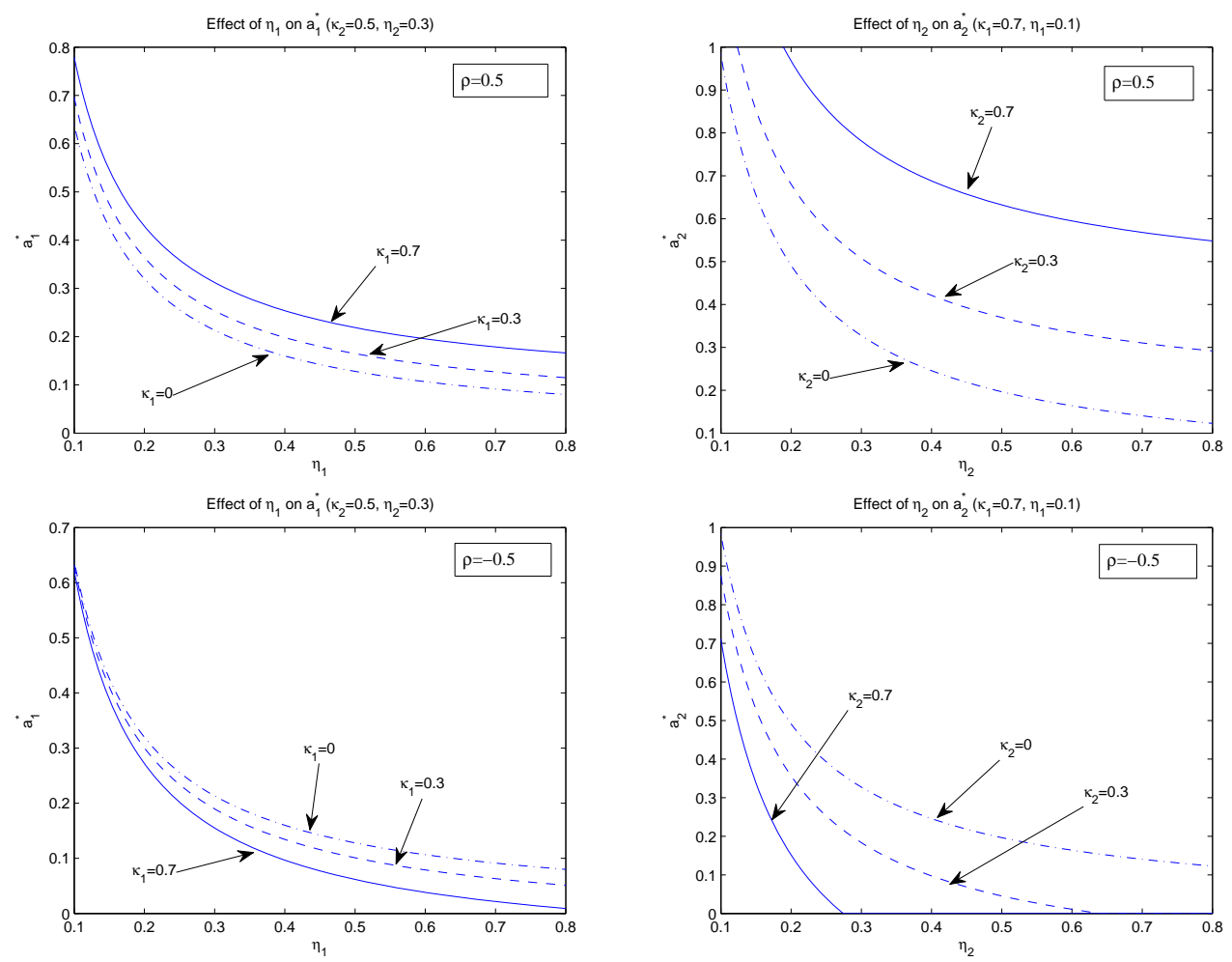

Figure 1: Effect of $\eta_{k}$ on $a_{k}^{*}(0)$, for $k=1,2$.

Note: The figures on the top row show the effect of $\eta_{k}$ on $a_{k}^{*}(0)$, for $k=1,2$, when $\rho=0.5$, whereas the figures on the bottom row show the effect of $\eta_{k}$ on $a_{k}^{*}(0)$, for $k=1,2$, when $\rho=-0.5$.

Figure 2 shows the effects of the rate of return $\left(m_{S}\right)$ and the volatility $\left(\sigma_{S}\right)$ of the risky asset $S$ on the investment strategy of insurer $k$ at equilibrium. The top row of Figure 2 captures the linear relationship between $b_{k}^{*}(0)$ and $m_{S}$, which is in agreement with the representation of $b_{k}^{*}(0)$ in (47). Increasing the rate of return of the risky asset $S$ increases the amount invested in $S$ for a given value of $\sigma_{S}$. The bottom row of Figure 2 also shows that $\sigma_{S}$ and $b_{k}^{*}(0)$ are inversely proportional to each other. This makes sense because high volatility in the risky asset $S$, makes it more likely that the risky asset will drop in value, thereby eroding the terminal value of each insurer. Collectively, Figure 2 serves as a visual confirmation that holding the risky asset $S$ increases as the Sharpe ratio $\mathcal{S}(0)$ increases.

Similar to the case of the proportional reinsurance strategy at equilibrium, the concept of competition can also be found in Figure 2. In particular, increasing the dependence parameter $\kappa_{k}$ increases the amount invested in the risky asset $S$, provided $m_{S} \geq r$. This is in line with our discussion on the the proportional reinsurance strategy $a_{k}^{*}(0)$ at equilibrium, increasing the amount invested in risky asset $S$ increases the chance of generating greater terminal wealth against that of his competitor at the terminal time $T$, if $m_{S} \geq r$, but off-set by a high value of $\sigma_{S}$. 

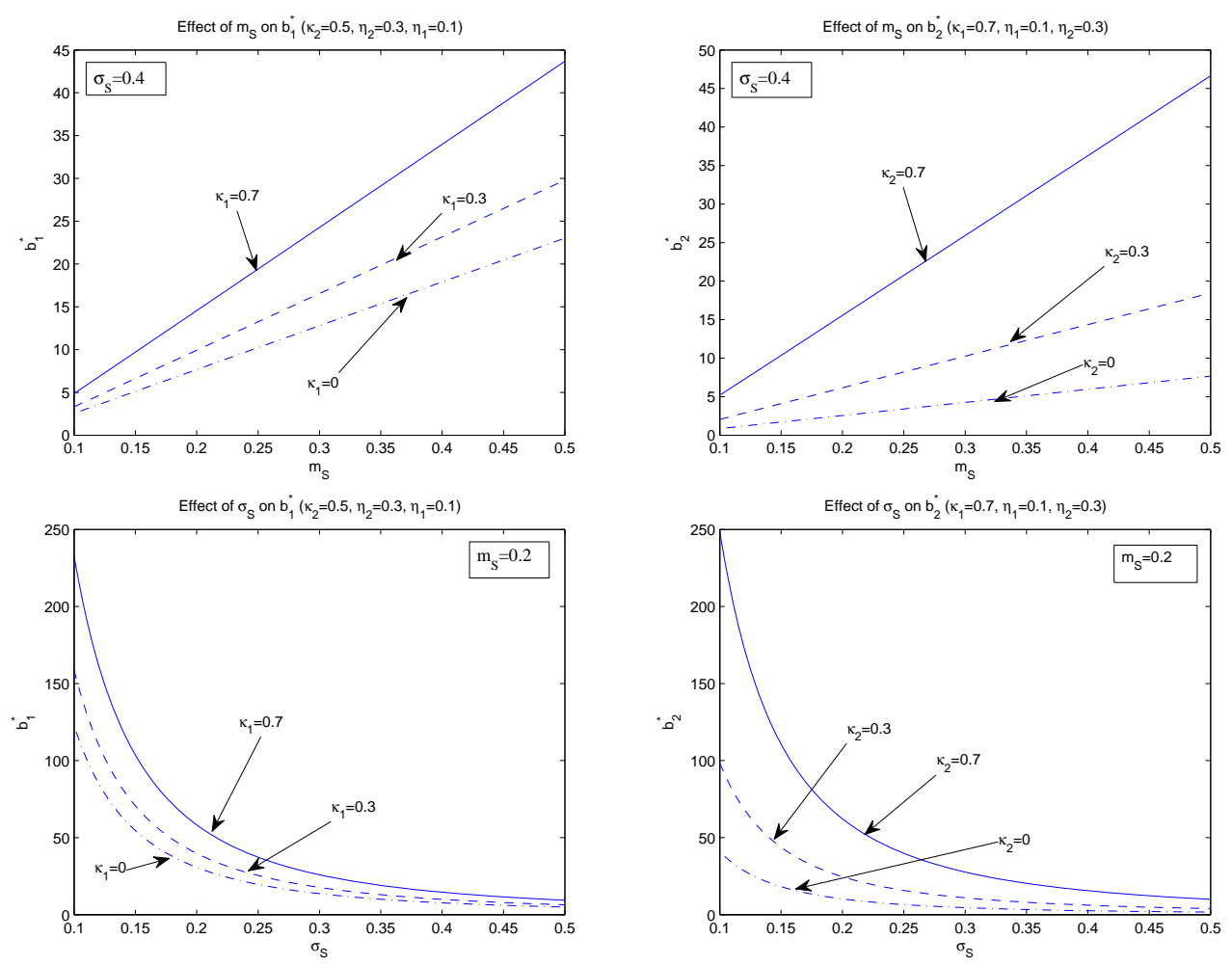

Figure 2: Effects of $m_{S}$ and $\sigma_{S}$ on $b_{k}^{*}(0)$, for $k=1,2$.

Note: The figures on the top row show the effect of $m_{S}$ on $b_{k}^{*}(0)$, for $k=1,2$, when $\sigma_{S}=0.4$, whereas the figures on the bottom row show the effect of $\sigma_{S}$ on $b_{k}^{*}(0)$, for $k=1,2$, when $m_{S}=0.2$.

\section{Conclusion}

In this paper, we consider the relative performance of two insurance companies in the form of a non-zero sum stochastic differential game framework. More specifically, we study the problem in which each insurer has the option of purchasing reinsurance protection and investing in the stock market. Similar to Espinosa and Touzi (2013), we incorporate the concept of competition into the problem by assuming that each insurer will make an optimal proportional reinsurance and investment decision, such that his expected utility of the difference between his terminal wealth and that of his competitor is maximized. To capture the effect of economic cycles on the optimal decisions of the insurance companies, we assume that the coefficients of each insurer's surplus process are modulated by a continuous-time Markov chain and an independent market index processes. Sufficient conditions for the proportional reinsurance and investment strategies to exist at equilibrium are provided. In the case of the exponential utility function, we obtain the closed-form solutions to the proportional reinsurance and investment strategy, together with the corresponding value function, for each insurer at equilibrium.

The present paper only covers the beginning of the optimal decision in the presence of competition. The non-zero sum game problem considered in this paper can be extended in many directions. For example, it would be interesting to study the problem under the possibility 
of investing multiple assets, which is more in line with reality. However, as shown in the case of multi-asset strategic investment with no competition, the condition of optimality is by no means trivial, as we find ourselves working under the multi-dimensional optimization problem. Explicit solutions to such optimization problems can be of great importance in showing the existence of a Nash equilibrium. In addition, instead of considering one competitor, we could embrace the ideas of Espinosa and Touzi (2013) to study the difference between one insurance company's wealth and the average of other insurance companies. The benefit of this approach is that it allows us to endogenize the concept of a benchmark process that gauges the performance of an insurance company against its peers. Finally, as presented in Theorem 4.2 , the optimal terminal value functions and equilibrium strategies for the case of the general utility functions are the solutions to the coupled PDEs. When closed-form solutions are not available, numerical approximation becomes inevitable. The issues of convergence and stability for any proposed algorithm would be of great interest.

\section{Acknowledgements}

The authors thank the referees for their thoughtful comments and their efforts towards improving the manuscript. Alain Bensoussan acknowledges the financial support from the Hong Kong RGC GRF 500111. Chi Chung Siu acknowledges the financial support from the Quantitative Finance Research Center (QFRC) at the University of Technology, Sydney. Phillip Yam acknowledges the financial support from the Hong Kong RGC GRF 404012 for the project titled Advanced Topics In Multivariate Risk Management In Finance And Insurance, The Chinese University of Hong Kong Direct Grant 2010/2011 Project ID: 2060422, and The Chinese University of Hong Kong Direct Grant 2011/2012 Project ID: 2060444. Phillip Yam also expresses his sincere gratitude to both Hausdorff Center for Mathematics of the University of Bonn and Mathematisches Forschungsinstitut Oberwolfach (MFO) in the German Black Forest for their hospitality during the preparation of the present work. Hailiang Yang would like to thank Research Grants Council of the Hong Kong Special Administration Region (project No: HKU 706611P) for their support. Finally, the authors are grateful to K. C. Sung, a former student of Phillip Yam and Hailiang Yang, for the preliminary discussion on the BSDE approach to the reinsurance-only game problem.

\section{References}

[1] Bäuerle, N., Rieder, U. (2004). Portfolio optimization with Markov-modulated stock prices and interest rates. IEEE Transactions on Automatic Control 49, 442-447.

[2] Bensoussan, A., Frehse J. (2000). Stochastic games for N players. Journal of Optimization Theory and Applications 105(3), 543-565.

[3] Browne, S. (1995) Optimal investment policies for a firm with a random risk process: exponential utility and minimizing the probability of ruin. Mathematics of Operations Research 20 (4) 937-958. 
[4] Browne, S. (2000). Stochastic differential portfolio games. Journal of Applied Probability $37,126-147$.

[5] Bühlmann, H. (1970). Mathematical Methods in Risk Theory. Springer, Berlin.

[6] Busch, M., Korn, R., Seifried, F. T. (2013). Optimal consumption and investment for a large investor: An intensity-based control framework. Mathematical Finance 23(4), $687-717$.

[7] Çanakoğlu, E., Özekici, S. (2010). Portfolio selection in stochastic markets with HARA utility functions. European Journal of Operational Research 201(2), 520-536.

[8] Chen, P., Yam, S. C. P. (2013). Optimal proportional reinsurance and investment with regime-switching for mean-variance insurers. Insurance: Mathematics and Economics 53, 871-883.

[9] Elliott, R. J. (1976). The existence of value in stochastic differential games. SIAM Journal of Control and Optimization 14(1), 85-94.

[10] Elliott, R. J., Aggoun, L., Moore, J. B (1994). Hidden Markov Models: Estimation and Control. Springer, Berlin.

[11] Elliott, R. J., Siu, T. K. (2011a). A BSDE approach to a risk-based optimal investment of an insurer. Automatica 47 (2), 253-261.

[12] Elliott, R. J., Siu, T. K. (2011b). A stochastic differential game for optimal investment of an insurer with regime switching. Quantitative Finance 11 (3), 365-380.

[13] Elliott, R. J., Siu, T.K., Yang, H. (2010). Filtering a Markov modulated random measure. IEEE Transactions on Automatic Control 55 (1), 74-88.

[14] Espinosa, G.-E., Touzi, N. (2013). Optimal investment under relative performance concerns. To appear: Mathematical Finance.

[15] Ferguson, T. S. (1965). Betting systems which minimize the probability of ruin. SIAM Journal of Applied Mathematics 13, 795-818.

[16] Gerber, H. (1970). Mathematical Methods in Risk Theory. Springer, Berlin.

[17] Liu, Y., Ma, J. (2009). Optimal reinsurance/investment problems for general insurance models. The Annals of Applied Probability 19 (4), 1495-1528.

[18] Klugman, S. A., Panjer, H. H., Willmot, G. E. (2008). Loss Models. John Wiley, Hoboken, N. J.

[19] Ng, A. C. Y., Yang, H. (2006). On the joint distribution of surplus prior and immediately after ruin under a Markovian regime switching model. Stochastic Processes and Their Applications 116 (2), 244-266.

[20] Øksendal, B. and Sulem, A. (2011). Portfolio optimization under model uncertainty and BSDE games. Quantitative Finance 11 (11), 1665-1674. 
[21] Pham, H. (2009). Continuous-time Stochastic Control and Optimization with Financial Applications. Springer, Berlin.

[22] Sotomayor, L. R., Cadenillas, A. (2009). Explicit solutions of consumption-investment problems in financial markets with regime switching. Mathematical Finance 19(2), 251279.

[23] Wang, G., Yu, Z. (2012). A partial information non-zero sum differential game of backward stochastic differential equations with applications. Automatica 48, 342-352.

[24] Yang, H., and Zhang, L. (2005). Optimal investment for insurer with jump-diffusion risk process. Insurance: Mathematics and Economics 37, 615-634.

[25] Zeng, X. (2010). A stochastic differential reinsurance game. Journal of Applied Probability 47 (3), 335-349.

[26] Zhou, X. Y., Yin, G. (2003). Markowitz's mean-variance portfolio selection with regime switching: A continuous-time model. SIAM Journal on Control and Optimization 42 (4), 1466-1482.

[27] Zhu, C. (2011). Optimal control of the risk process in a regime-switching environment. Automatica 47 (8), 1570-1579.

[28] Zhu, J., Yang, H. (2009). On differentiability of ruin functions under Markov-modulated model. Stochastic Processes and Their Applications 119 (5), 1673-1695. 